Pacific Journal of Mathematics

ON THE DECOMPOSITION OF REDUCIBLE PRINCIPAl
SERIES REPRESENTATION OF $p$-ADIT CHEVALLEY
GROUPS

Charles David Keys 


\title{
ON THE DECOMPOSITION OF REDUCIBLE PRINCIPAL SERIES REPRESENTATIONS OF $P$-ADIC CHEVALLEY GROUPS
}

\author{
Charles David Keys
}

\begin{abstract}
In this paper we study the decomposition of principal series representations of $p$-adic Chevalley groups which are induced from a minimal parabolic subgroup, and determine the structure of the commuting algebras of these representations.
\end{abstract}

\section{TABLE OF CONTENTS}

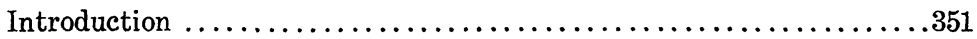

Chapter I. Intertwining Operators and the Commuting Algebra..357

1. The intertwining operators $A(w, \lambda)$ and $\mathfrak{a}(w, \lambda) \ldots 357$

2. The cocycle condition for $a(w, \lambda) \ldots \ldots \ldots \ldots . \ldots 361$

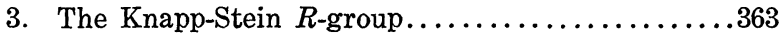

Chapter II. Classification of the $R$-groups $\ldots \ldots \ldots \ldots \ldots \ldots \ldots \ldots \ldots \ldots$

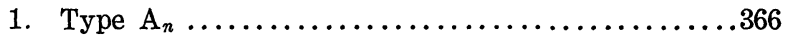

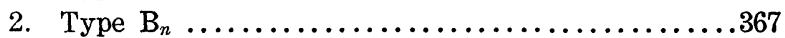

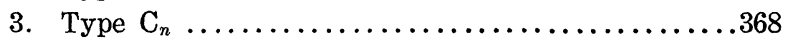

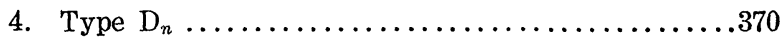

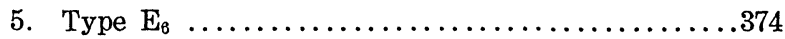

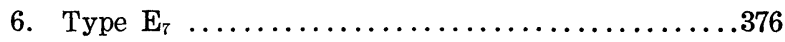

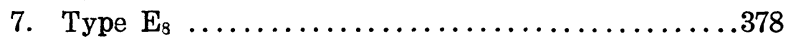

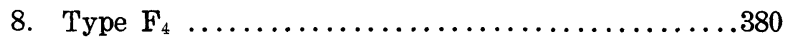

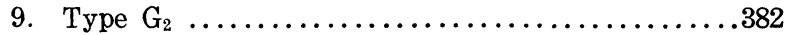

Chapter III. On the Decomposition of $\operatorname{Ind}_{B}^{G} \lambda \ldots \ldots \ldots \ldots \ldots \ldots . \ldots 383$

1. Multiplicities of the irreducible components......383

2. Some analysis on $L^{2}(V) \ldots \ldots \ldots \ldots \ldots \ldots \ldots \ldots \ldots$

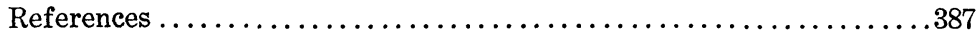

Introduction. Let $G$ be a split reductive $p$-adic group, $T$ a maximal split torus of $G$ and $B=T U$ a minimal parabolic subgroup of $G$. A (unitary) character $\lambda$ of $T$ may be extended trivially across $U$ to define a character of $B$. The induced representation $\operatorname{Ind}_{B}^{G} \lambda$ is called a (unitary) principal series representation of $G$.

Let $W$ be the Weyl group of $G$ and choose $w \in W$. Then the representations $\operatorname{Ind}_{B}^{G} \lambda$ and $\operatorname{Ind}_{B}^{G} w \lambda$ are equivalent. The problem of constructing explicit intertwining operators $\mathfrak{a}(w, \lambda)$ between $\operatorname{Ind}_{B}^{G} \lambda$ and $\operatorname{Ind}_{B}^{G} w \lambda$ has been studied for real semi-simple Lie groups by Kunze and Stein [24, 25, 26] Schiffmann [30], Knapp [14, 15, 16] Knapp and Stein $[17,18,19,20,21,22]$ Harish-Chandra [10] and others. For groups defined over a $p$-adic filed $\mathfrak{k}$, these operators were first studied for SL(2) by Sally [28], and then for $p$-adic Chevalley groups by Winarsky [36, 37], who used them to determine necessary and 
sufficient conditions for $\operatorname{Ind}_{B}^{G} \lambda$ to be reducible. A more general study of intertwining operators for $p$-adic groups has been carried out by Harish-Chandra, Silberger and others.

Let $W_{\lambda}=\{w \in W \mid w \lambda=\lambda\}$. By Bruhat theory [32], the length of the composition series of $\operatorname{Ind}_{B}^{G} \lambda$ is bounded by $\left|W_{\lambda}\right|$. Thus $\operatorname{Ind}_{B}^{G} \lambda$ is irreducible if $\lambda$ is a nonsingular character of $T$, i.e., $W_{\lambda}=\{1\}$.

Suppose that $\lambda$ is a singular character of $T$ and that $w \lambda=\lambda$, $1 \neq w \in W$. Then $\mathfrak{a}(w, \lambda)$ is an intertwining operator for $\operatorname{Ind}_{B}^{G} \lambda$ which may or may not be scalar. By an unpublished theorem of HarishChandra, the operators $\left\{a(w, \lambda) \mid w \in W_{\lambda}\right\}$ span the commuting algebra $C(\lambda)$ of $\operatorname{Ind}_{B}^{G} \lambda$. However, these operators may not be distinct.

We determine a basis for $C(\lambda)$ consisting of a subgroup of these operators. Following Knapp and Stein $[14,19]$, we write $W_{\lambda}=$ $R \ltimes W^{\prime}$ as a semi-direct product, with $W^{\prime}=\left\{w \in W_{\lambda} \mid \mathfrak{a}(w, \lambda)\right.$ is scalar $\}$. We show that, with appropriate normalizations, a cocycle condition holds and that $w \mapsto \mathfrak{a}(w, \lambda)$ is a homomorphism from $W_{\lambda}$ to the group of invertible intertwining operators for $\operatorname{Ind}_{B}^{G} \lambda$. We then give an elementary proof that the operators $\{a(w, \lambda) \mid w \in R\}$ are linearly independent. This is essentially Silberger's theorem [33] for the case of minimal parabolics. These facts combined with HarishChandra's theorem imply that $\{\mathfrak{a}(w, \lambda) \mid w \in R\}$ is a basis of the commuting algebra $C(\lambda)$, and further, that $C(\lambda)$ is isomorphic to the group algebra $C[R]$.

For complex groups, $\operatorname{Ind}_{B}^{G} \lambda$ is always irreducible.

Knapp, in collaboration with Stein, $[15,16]$ has shown that for real groups, $R \cong \boldsymbol{Z}_{2} \times \cdots \times \boldsymbol{Z}_{2}$ with the number of factors of $\boldsymbol{Z}_{2}$ bounded by the dimension of $T$. Thus $\operatorname{Ind}_{B}^{G} \lambda$ decomposes into $|R|$ components, each occuring with multiplicity one.

For $p$-adic groups, $\operatorname{Ind}_{B}^{G} \lambda$ does not always decompose simply. We classify the nontrivial $R$-groups which occur.

Type $\mathrm{A}_{n} . \quad R$ is abelian and $|R|$ divides $n+1$. If the largest

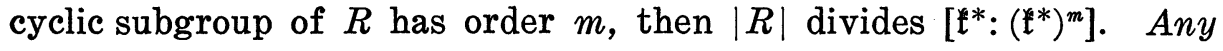
finite abelian group with these properties occurs as an $R$-group.

Type $\mathrm{B}_{n} . \quad R \cong Z_{2} \times \cdots \times Z_{2}$ and $|R|$ divides both $2 n$ and $\left[\mathfrak{l}^{*}:\left(\mathfrak{l}^{*}\right)^{2}\right]$.

Type $\mathrm{C}_{n} . \quad R \cong \boldsymbol{Z}_{2} \times \cdots \times \boldsymbol{Z}_{2}$ with the number of factors of $\boldsymbol{Z}_{2}$ bounded by $n$ and $\left[\mathfrak{l}^{*}:\left(\mathfrak{k}^{*}\right)^{2}\right]-1$.

Type $\mathrm{D}_{n}$. $R$ may be nonabelian. (This general fact was first discovered by Knapp and Zuckerman.)

(a) Suppose $n$ even. Then if $R$ is abelian, $R \cong \boldsymbol{Z}_{2} \times \cdots \times \boldsymbol{Z}_{2}$ with the number of factors bounded by $n-1$ and by $\left[\mathfrak{t}^{*}:\left(\mathfrak{k}^{*}\right)^{2}\right]-1$. 
If $R$ is nonabelian, $\boldsymbol{R} \cong\left(\boldsymbol{Z}_{2} \times \cdots \times \boldsymbol{Z}_{2}\right) \ltimes\left(\boldsymbol{Z}_{2} \times \boldsymbol{Z}_{2} \times \cdots \times \boldsymbol{Z}_{2}\right)$ with the order of the first factor dividing both $2 n$ and $\left[\mathfrak{k}^{*}:\left(\mathfrak{f}^{*}\right)^{2}\right]$ and the number of factors of $\boldsymbol{Z}_{2}$ in the normal subgroup an odd number bounded by $n-1$ and by $\left[\mathfrak{f}^{*}:\left(\mathfrak{f}^{*}\right)^{2}\right]-1$.

(b) Suppose $n$ is odd. Then if $R$ is abelian, $R \cong \boldsymbol{Z}_{2} \times \cdots \times \boldsymbol{Z}_{2}$ with the number of factors bounded by $n-1$ and $\left[\mathfrak{t}^{*}:\left(\mathfrak{l}^{*}\right)^{2}\right]-1$, or $R \cong \boldsymbol{Z}_{4}$. If $R$ is nonabelian, then $R \cong \boldsymbol{Z}_{4} \ltimes\left(\boldsymbol{Z}_{2} \times \cdots \times \boldsymbol{Z}_{2}\right)$ with the number of factors of $\boldsymbol{Z}_{2}$ in the normal subgroup an even number bounded by $n-3$ and $\left[\mathfrak{f}^{*}:\left(\mathfrak{k}^{*}\right)^{2}\right]-2$.

Type $\mathrm{E}_{6} . \quad R \cong 1, \boldsymbol{Z}_{2}, \boldsymbol{Z}_{3}, \boldsymbol{Z}_{3} \times \boldsymbol{Z}_{3}$ or $\boldsymbol{Z}_{6}$. Further, $\boldsymbol{Z}_{3} \times \boldsymbol{Z}_{3}$ can occur if and only if $p=3$ or 3 divides $q-1$.

Type $\mathrm{E}_{7} . \quad R$ may be nonabelian. If so, $R \cong$ dihedral group $D$ of order 8 , or $R \cong D \times Z_{2} . \quad D \times Z_{2}$ can occur if and only if $p=2$ or 4 divides $q-1$.

If $R$ is abelian, then $R \cong \boldsymbol{Z}_{2}^{n}$ with $0 \leqq n \leqq 4, \boldsymbol{Z}_{3}, \boldsymbol{Z}_{4}$, or $\boldsymbol{Z}_{6} . \boldsymbol{Z}_{2}^{n}$ will occur if and only if $\left[k^{*}:\left(k^{*}\right)^{2}\right] \geqq 2^{n}, 0 \leqq n \leqq 4$. $Z_{4}$ occurs if and only if $p=2$. $Z_{3}$ and $Z_{6}$ occur if and only if $p=3$ or 3 divides $q-1$.

Type $\mathrm{E}_{8} . \quad R$ may be nonabelian. All nonabelian $R$ are conjugate. The nonabelian $R$-group will occur if and only if $\left[k^{*}:\left(k^{*}\right)^{2}\right] \geqq$ 16. It has order 128 , has 65 conjugacy classes, and $R \bmod \left\langle w_{0}\right\rangle$ is abelian.

If $R$ is abelian, then $R \cong \boldsymbol{Z}_{2}^{n}$ with $0 \leqq n \leqq 4, \boldsymbol{Z}_{4}, \boldsymbol{Z}_{4} \times \boldsymbol{Z}_{2}, \boldsymbol{Z}_{3}$, $\boldsymbol{Z}_{3} \times \boldsymbol{Z}_{3}$, or $\boldsymbol{Z}_{5}$. $\quad \boldsymbol{Z}_{2}^{n}$ occurs if and only if $\left[k^{*}:\left(k^{*}\right)^{2}\right] \geqq 2^{n+1}, 0 \leqq n \leqq 4$. $\boldsymbol{Z}_{4}$ occurs if and only if $p=2$ or 4 divides $q-1$. $\boldsymbol{Z}_{4} \times \boldsymbol{Z}_{2}$ occurs if and only if $p=2 . \quad Z_{3}^{n}$ occurs if and only if $\left[k^{*}:\left(k^{*}\right)^{3}\right] \geqq 3^{n+1}, n=1$ or 2 . $Z_{5}$ occurs if and only if $\left[k^{*}:\left(k^{*}\right)^{5}\right] \geqq 25$.

Type $\mathrm{F}_{4} . \quad R \cong \boldsymbol{Z}_{2}$ or $\boldsymbol{Z}_{3} . \quad \boldsymbol{Z}_{3}$ can occur as $R$-group if and only if $p=3$ or 3 divides $q-1$.

Type $\mathrm{G}_{2} . \quad R \cong \boldsymbol{Z}_{2}$.

The order of $R$ depends on $n$ and on the arithmetic of the field $f$, i.e., on the existence of enough multiplicative characters of order 2 , or of order dividing $n+1$ in the case of type $A_{n}$ and of order 3 in the case of type $\mathrm{F}_{4}$.

We note that the methods in this paper also apply to Chevalley groups defined over the reals $\boldsymbol{R}$ and the complex numbers $\boldsymbol{C}$. Since $C^{*}$ has no nontrivial characters of finite order, $R=\{1\}$ and thus $\operatorname{Ind}_{B}^{G} \lambda$ is irreducible for Chevalley groups over $C$. Since $\boldsymbol{R}^{*}$ has only 
one nontrivial character of finite order, we can recover the KnappStein result for Chevalley groups over $\boldsymbol{R}$. Further $R \cong \boldsymbol{Z}_{2}$ or $\{1\}$ except in the case of $D_{n}, n$ even, for which $R \cong \boldsymbol{Z}_{2} \times \boldsymbol{Z}_{2}$ can occur [19].

The organization of this paper is as follows. We establish notation and definitions in a preliminary section. In $\S 1$ of Chapter 1 we study the normalization and analytic continuation of the intertwining operators $A(w, \lambda)$ and $a(w, \lambda)$ for Macdonald's "groups of p-adic type." In $\S 2$ we show that with appropriate normalizations the operators $\mathfrak{a}(w, \lambda)$ are well-defined and establish a cocycle relation for these operators with no condition on the lengths of the Weyl group elements. In $\S 3$ we follow Knapp [14, 15] to develop the theory of the $R$-group for $p$-adic Chevalley groups, and show that $C(\lambda)=C[R]$.

Chapter 2 is devoted to the classification of $R$-groups. In each section, we explicitly determine all $R$ which occur for one type of root system, by constructing a list of $\lambda$ and $R$ and showing that every nontrivial $R$-group is conjugate to one on the list.

In Chapter 3 we use the intertwining operators to study the problem of decomposing $\operatorname{Ind}_{B}^{G} \lambda$ into irreducible components in a "Fourier transform realization" on $L^{2}(V)$, where $V$ is the unipotent radical of the Borel subgroup opposed to $B$. A class of functions is found on which $a(w, \lambda)$ acts as multiplication by a function $M(w, \lambda)$ and we show that the operators $\{\mathfrak{a}(w, \lambda) \mid w \in R\}$ are linearly independent.

Most of these results appeared in the author's thesis. I would like to express my gratitude and thanks to my advisor, Professor Paul J. Sally, Jr., for his help and guidance.

With some restrictions on the residual characteristic of $k$, independent work of Müller gives partial results describing the $R$-groups which occur for the classical Chevalley groups. See "Integrales d'entrelacement pour un groupe de Chevalley sur un corps $p$-adique" in the Springer Lecture Notes 739.

Preliminaries and definitions. Let $\mathfrak{t}$ be a nonArchimedean local field. We will be concerned mainly with Chevalley groups $G$ defined over $\mathfrak{f}$, although some of our results will apply to the of any reductive algebraic group defined over $\mathfrak{t}$.

Let $d x$ be Haar measure on $\mathfrak{k}$ and || the absolute value on defined by $d(a x)=|a| d x$.

Let $\mathcal{O}=\{x \in \mathfrak{t}|| x \mid \leqq 1\}$ be the ring of integers of $\mathfrak{k}, \Pi$ a prime element of $\mathscr{O}$, and $\mathfrak{p}=\{x \in \mathfrak{f}|| x \mid<1\}$ the unique nonzero prime ideal of $\mathscr{O}$. Then $\mathscr{O} / \mathfrak{p}$ is a finite field with $q$ elements, where $q$ is a prime power. 
Normalize Haar measure on $\mathfrak{f}$ so that volume $(\mathscr{O})=1$. Then $\mathfrak{p}^{n}=\left\{x \in \mathfrak{f}|| x \mid \leqq q^{-n}\right\}$ has volume $q^{-1}$. The collection $\mathfrak{p}^{n}, n \in Z$, forms a fundamental system of neighborhoods at 0 for the topology on $\mathfrak{k}$, which are both open and compact. Thus is totally disconnected.

Haar measure on $\mathfrak{k}^{*}$ is $d^{*} x=|x|^{-1} d x$.

Let $U_{0}=U=\mathscr{O}^{*}=\{x \in \mathscr{O}|| x \mid=1\}$ be the units in $\mathcal{O}$. For each positive integer $n$, set $U_{n}=1+\mathfrak{p}^{n}$. Then the collection $U_{n}$ forms a fundamental system of neighborhoods at 1 for $\mathfrak{k}^{*}$ consisting of compact and open subgroups.

The additive group of $\mathfrak{t}$ is self-dual. Fix a nontrivial additive character $\chi$ of $\mathfrak{t}$. Then any character of $\mathfrak{t}$ is of the form $\chi_{a}(x)=$ $\chi(a x)$. Define the conductor cond $(\chi)$ of $\chi$ to be $n$ if $\chi$ is trivial on $\mathfrak{p}^{n}$ and nontrivial on $\mathfrak{p}^{n-1}$.

Since any $x \in \mathbb{1}^{*}$ may be written as $x=\Pi^{n} u, n \in Z, n \in U$, we see that $\mathfrak{t}^{*} \cong Z \times U$. Thus $\left(\mathfrak{t}^{*}\right)^{\wedge} \cong Z^{\wedge} \times U^{\wedge}$ and any character of $\mathfrak{k}^{*}$ is given by $\lambda\left(\Pi^{n} u\right)=\left|\Pi^{n}\right|^{s} \lambda^{*}(u)$ where $s \in C, \operatorname{Re} s=0$, and $\lambda^{*}$ is the restriction of $\lambda$ to the compact group $U$. We obtain quasicharacters of $\mathfrak{k}^{*}$ by $\lambda\left(\Pi^{n} u\right)=\left|\Pi^{n}\right|^{s} \lambda^{*}(u)$ where $s \in C$. Define $\operatorname{Re} \lambda=$ $\operatorname{Re}(s) . \quad \lambda$ is unramified if $\lambda^{*}=1$. Otherwise $\lambda$ is ramified. Define $\operatorname{deg}(\lambda)=n$ if $\lambda$ is trivial on $U_{n}$ but nontrivial on $U_{n-1}$.

A gamma function $\Gamma(\lambda)$ is associated to each nontrivial multiplicative quasi-character $\lambda[29,35]$. If $\lambda=|\cdot|{ }^{s} \lambda^{*}$ is ramified of degree $h$, then $\Gamma(\lambda)=P \cdot V \cdot \int \bar{\chi}(x) \lambda(x)|x|^{-1} d x=c_{\lambda^{*}} q^{h(8-1 / 2)}$, where $\left|c_{\lambda^{*}}\right|=1$ and $c_{\lambda^{*}} c_{\lambda^{*}-1}=\lambda^{*}(-1)$. If $\lambda=|\cdot|^{s}$ is unramified, then $\Gamma(\lambda)=$ $P \cdot V \cdot \int \bar{\chi}(x)|x|^{s-1} d x=\left(1-q^{s-1}\right) /\left(1-q^{-s}\right)$ if $\operatorname{Re} \lambda>0$, and is the analytic continuation of this function into the left half-plane for $\operatorname{Re} \lambda \leqq 0$, $s \neq 0$.

Let $G$ be a Chevalley group over $\mathfrak{f}$ [34]. Let $L$ be the semisimple Lie algebra over $C$ which determines $G$ and $\underline{h}$ a Cartan subalgebra. Then $L=\underline{h} \oplus \sum_{\alpha \neq 0} L_{\alpha}$ where $\alpha$ is a root. Denote the set of roots by $\Phi$.

Let $w_{\alpha}$ denote the reflection in the hyperplane orthogonal to $\alpha$ in the Euclidean space $Z[\Phi] \otimes \boldsymbol{R}$ and let the Weyl group $W$ be the group generated by the $w_{\alpha}, \alpha \in \Phi$.

$G$ is generated by subgroups $U_{\alpha}=\left\{x_{\alpha}(t) \mid t \in \mathfrak{f}\right\}, \alpha \in \Phi . \quad U_{\alpha}$ carries a natural valuation $U_{\alpha+n}=\left\{x_{\alpha}(t) \mid t \in \mathfrak{p}^{n}\right\}$.

Let $w_{\alpha}(t)=x_{\alpha}(t) x_{-\alpha}\left(-t^{-1}\right) x_{\alpha}(t)$ and $h_{\alpha}(t)=w_{\alpha}(t) w_{\alpha}(1)^{-1}$ for $t \in \mathfrak{t}^{*}$. Let $T$ be the subgroup generated by all $h_{\alpha}(t), \alpha \in \Phi$. Then $W=$ $N(T) / T$ and $w_{\alpha}(t)$ is a coset representative in $N(T)$ for the reflection $w_{\alpha}$.

Fix an ordering on the root system $\Phi$. This determines a set of positive roots and a set of simple roots which forms a base for 
$\Phi$. Let $U$ be the subgroup generated by all $U_{\alpha}$, where $\alpha$ is a positive root.

Then $T$ is a maximal torus of $G$ and $B=T U$ is a Borel subgroup of $G$ with unipotent radical $U$.

For each root $\alpha$, there is a canonical homomorphism $\varphi_{\alpha}$ from SL $(2, \mathfrak{k})$ into the subgroup of $G$ generated by $U_{\alpha}$ and $U_{-\alpha}$ such that

$$
\begin{array}{ll}
\varphi_{\alpha}\left(\begin{array}{ll}
1 & t \\
0 & 1
\end{array}\right) & =x_{\alpha}(t), \quad \varphi_{\alpha}\left(\begin{array}{rl}
0 & 1 \\
-1 & 0
\end{array}\right)=w_{\alpha}(1), \\
\varphi_{\alpha}\left(\begin{array}{ll}
1 & 0 \\
t & 1
\end{array}\right) & =x_{-\alpha}(t), \quad \text { and } \quad \varphi_{\alpha}\left(\begin{array}{ll}
t & 0 \\
0 & t^{-1}
\end{array}\right)=h_{\alpha}(t) .
\end{array}
$$

The kernel of $\varphi_{\alpha}$ is either trivial or $\{ \pm I\}$.

If $\lambda$ is a character of $T$, we define for each root $\alpha$ a character $\lambda_{\alpha}$ of $\mathfrak{1}^{*}$ by $\lambda_{\alpha}(t)=\lambda\left(h_{\alpha}(t)\right)$. The Weyl group $W$ acts on $T$ and thus on characters of $T$. We note that $w \lambda_{\alpha}(t)=w \lambda\left(h_{\alpha}(t)\right)=\lambda\left(w^{-1} h_{\alpha}(t) w\right)=$ $\lambda\left(h_{w^{-1} \alpha}(t)\right)=\lambda_{w^{-1} \alpha}(t)$. The one-parameter subgroups $h_{\alpha}(t)$ form a root system $\Phi^{\curlyvee}$ dual to $\Phi$ in $\operatorname{Hom}\left(\mathfrak{k}^{*}, T\right) \otimes R$. $w$ acts on $\lambda_{\alpha}$ as $w$ acts on $\alpha^{v}$, as $w^{-1}$ acts on $\alpha$. We use this observation to simplify notation and calculations in Chapter 2.

Let $K$ be the subgroup of $G$ generated by $\left\{x_{\alpha}(t) \mid \alpha \in \Phi, t \in \mathcal{O}\right\}$. Then $K$ is a good maximal compact subgroup of $G$ [4. 27], and there is an Iwasawa decomposition $G=K B=K T U$, nonuniquely.

More generally, suppose $G$ is the group of $\mathfrak{l}$-rational points of a reductive algebraic group defined over $\mathfrak{k}$. A Borel subgroup $B$ is a maximal connected solvable subgroup of $G$. A parabolic subgroup $P$ is a subgroup of $G$ containing a Borel subgroup. Let $N$ be the

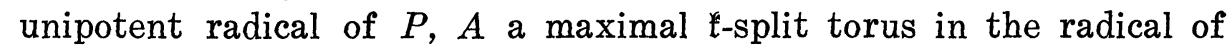
$P$ and $M=Z_{G}(A)$. Then $P$ has a Levi decomposition $P=M N$.

$B$ has Levi decomposition $T U$ where $T$ is the centralizer in $G$ of a maximal $\mathfrak{\ell}$-split torus $A$ of $G . \quad W=N(A) / Z(A)$ acts on $A$ and thus on Hom $\left(A, \mathfrak{k}^{*}\right)$, which is dually paired over $\boldsymbol{Z}$ with Hom $\left(\mathfrak{f}^{*}, A\right)$. If $G$ is semi-simple, the root system $\Phi=\Phi(G, A)$ spans Hom $\left(A, \mathfrak{t}^{*}\right) \otimes$ $\boldsymbol{R}$, and we have the dual root system $\Phi^{v}$ in $\operatorname{Hom}\left(\mathfrak{l}^{*}, A\right) \otimes \boldsymbol{R}$ [1].

Bruhat-Tits theory gives a generating set of valuated root data and the existence of good maximal compact subgroups of $G$, for which Iwasawa and Cartan decompositions hold [4, 27].

A topological group $G$ is said to be totally disconnected (t.d.) if there exists a neighborhood basis at 1 for the topology on $G$ consisting of open compact subgroups. A function on a t.d. group is smooth, or $C^{\infty}$, if it is locally constant.

Let $G$ be a t.d. group and $V$ a vector space over $C$. A representation $(\Pi, V)$ of $G$ is a mapping $\Pi: G \rightarrow$ End $(V)$ such that $\Pi(1)=1$ and $\Pi(x y)=\Pi(x) \Pi(y)$ for all $x, y \in G$. A vector $v \in V$ is smooth if 
$x \mapsto \Pi(x) v$ is a smooth function on $G$. We say that $\Pi$ is smooth if every $v \in V$ is smooth.

If $H$ is a subgroup of $G$, define $V^{I I}=\{v \in V \mid \Pi(h) v=v$ for all $h \in H$ \}. A representation $(I, V)$ of $G$ is admissible if $\Pi$ is smooth and $\operatorname{dim} V^{H}<\infty$ for any open subgroup $H$ of $G$.

A subspace $W$ of $V$ is invariant if $\Pi(x) W=W$ for all $x \in G$. The representation $(\Pi, V)$ is (algebraically) irreducible if $V$ has no nontrivial invariant subspaces.

$(\Pi, V)$ is a pre-unitary representation if there is a positivedefinite hermitian form on $V$ which is preserved by all $\Pi(x), x \in G$. We may take the completion of $V$ with respect to the inner product defined by this form to obtain a unitary representation of $G$ on a Hilbert space $\mathscr{H}$, of which $V$ is the subspace of smooth vectors.

We also require that $x \mapsto \Pi(x)$ be continuous for unitary representations. $(\Pi, \mathscr{C})$ is irreducible if there are no nontrivial closed invariant subspaces.

Let $(\Pi, V)$ and $\left(\Pi^{\prime}, V^{\prime}\right)$ be representations of $G$. An intertwining operator between $I I$ and $\Pi^{\prime}$ is a linear map $A: V \rightarrow V^{\prime}$ with the property that $A \Pi(x)=\Pi^{\prime}(x) A$ for all $x \in G . \quad \Pi$ is equivalent to $\Pi^{\prime}$ if $A$ can be chosen to be a bijection.

Define the commuting algebra of $(\Pi, V)$ to be $\{A: V \rightarrow V \mid A \Pi(x)=$ $\Pi(x) A$ for all $x \in G\}$.

If $\pi, \pi^{\prime}$ are unitary, we require an intertwining operator $A$ to be a bounded linear operator. $\pi$ and $\pi^{\prime}$ are (unitarily) equivalent if $A$ can be chosen to be a unitary operator.

We will use the following criterion for reducibility.

THeOREM. Suppose $(\pi, V)$ is a unitary representation of $G$. Then $\pi$ is irreducible if and only if its commuting algebra is onedimensional [32].

More detailed introductions to the representation theory of t.d. groups may be found in $[6,11,13,32]$.

\section{CHAPTER I}

\section{INTERTWINING OPERATORS AND THE COMMUTING ALGEBRA}

1. The intertwining operators $A(w, \lambda)$ and $\mathfrak{a}(w, \lambda)$. Let $P=$ $M N$ be a parabolic subgroup of $G$ and $(\sigma, V)$ an admissible representation of $M$, extended trivially across $N$. Define the representation $\operatorname{Ind}_{P}^{G} \sigma$ to be left translation in the space of functions $H_{\sigma}=\{f: G \rightarrow$ $V \mid f$ is locally constant and $f(g m n)=\delta_{P}^{-1 / 2} \sigma^{-1}(m) f(g)$ for all $g \in G$, $m \in M$, and $n \in N\}$. Since $G=K P$ with $K$ compact, $\operatorname{Ind}_{P}^{G} \sigma$ is an admissible representation of $G$. The factor $\delta_{P}^{-1 / 2}$ is used so that unitary 
representations induce to unitary representations. One could also take functions which are square integrable $\bmod P$.

From Bruhat theory, one knows that $\operatorname{Ind}_{P}^{G} \sigma$ and $\operatorname{Ind}_{P_{1}}^{G} \sigma_{1}$ have no composition factors in common if $P$ and $P_{1}$ are not conjugate in G. Further, Ind ${ }_{P}^{G} \sigma$ and $\operatorname{Ind}_{P}^{G} \sigma_{1}$ have a composition factor in common only if there exists a $w \in W$ normalizing $M$ such that $w \sigma$ is equivalent to $\sigma_{1}$. In this case, $\operatorname{Ind}_{P}^{G} \sigma$ is equivalent to $\operatorname{Ind}_{P}^{G} w \sigma$.

Jacquet's theorem states that any irreducible representation of a reductive $p$-adic group $G$ is a subrepresentation of $\operatorname{Ind}_{P}^{G} \sigma$ for some parabolic subgroup $P$, where $\sigma$ is a supercuspidal representation of $M[13,32]$.

Thus to give a complete list of the irreducible representations of $G$, one needs to decompose all $\operatorname{Ind}_{P}^{G} \sigma$, with equivalent factors arising only in the case of the equivalent representations $\operatorname{Ind}_{P}^{G} \sigma$ and $\operatorname{Ind}_{P}^{G} w \sigma$.

We study the problem of decomposing the representations $\operatorname{Ind}_{B}^{G} \lambda$, where $G$ is a Chevalley group over $\mathfrak{t}, B=T U$ is a Borel subgroup, and $\lambda$ is a (unitary) character of $T$.

Let $W_{\lambda}=\{w \in W \mid w \lambda=\lambda\}$ for $\lambda$ a quasi-character of $T$. By Bruhat theory, the length of the composition series of $\operatorname{Ind}_{B}^{G} \lambda$ is bounded by $\left|W_{\lambda}\right|$ if $\lambda$ is unitary.

Suppose $w \in W$. Intertwining operators $A(w, \lambda)$ between $\operatorname{Ind}_{B}^{G} \lambda$ and $\operatorname{Ind}_{B}^{G} w \lambda$ are defined initially for certain nonunitary $\lambda$. These operators are normalized to define operators $\mathfrak{a}(w, \lambda)$ which can be extended by analytic continuation to meromorphic functions in $\lambda$.

Fix a coset representative $\bar{w}$ in $N(T)$ for $w$. Define [30, 37]

$$
[A(\bar{w}, \lambda) f](g)=\int_{U \cap w \vee w^{-1}} f(g u \bar{w}) d u \text { for } f \in H_{\lambda} .
$$

We remark that if we choose a different coset representative $\bar{w}^{\prime}$ for $w$, then $\bar{w}^{-1} \bar{w}^{\prime} \in T$ and the operators differ by a scalar $\lambda^{-1} \delta_{B}^{-1 / 2}\left(\bar{w}^{-1} \bar{w}^{\prime}\right)$.

N. Winarsky has shown that $A(\bar{w}, \lambda) f(g)$ converges absolutely for quasi-characters $\lambda$ in the domain $D(w)=\left\{\lambda \mid \operatorname{Re} \lambda_{\alpha}>0\right.$ for $\left.\alpha \in R(w)\right\}$, where $R(w)=\{\alpha \in \Phi \mid \alpha>0$ and $w \alpha<0\}$, and that $A(\bar{w}, \lambda): H_{\lambda} \rightarrow H_{w \lambda}$ intertwines $\operatorname{Ind}_{B}^{G} \lambda$ and $\operatorname{Ind}_{B}^{G} w \lambda$. Further, if the condition $l\left(w^{\prime} w^{\prime \prime}\right)=$ $l\left(w^{\prime}\right)+l\left(w^{\prime \prime}\right)$ on lengths holds, then the cocycle condition $A\left(\bar{w}^{\prime} \bar{w}^{\prime \prime}, \lambda\right)=$ $A\left(\bar{w}^{\prime}, \bar{w}^{\prime \prime} \lambda\right) \circ A\left(\bar{w}^{\prime \prime}, \lambda\right)$ holds [37].

These results are true for $G$ a reductive $p$-adic group. The proofs are as in $[30,37]$ once we have the following.

LEMMA 1. Let $G$ be a reductive p-adic group. Let $\operatorname{Re} \lambda=|\lambda|$ and let $\chi_{\mathrm{Re} \lambda}$ be the $K$-fixed vector in $H_{\mathrm{Re} \lambda}$ defined by $\chi_{\mathrm{Re} \lambda}(k t u)=$ $\operatorname{Re}(\lambda)^{-1} \rho^{-1}(t)$. Suppose $\operatorname{Re} \lambda_{\alpha}>0$. Then 


$$
\int_{U_{\alpha}} \chi_{\mathrm{Re \lambda}}\left(u \bar{w}_{\alpha}\right) d u<\infty
$$

Proof. By Bruhat-Tits theory, the derived group of $G$ possesses a system of valuated root data, with properties which Macdonald has taken as axioms for a "group of $p$-adic type" [4, 27].

$B=T U$ is a minimal parabolic, where $T$ is now the centralizer of a maximal -split torus $A$ in $G$. There is a homomorphism $\nu$ with kernel $T \cap K$ from $N(A)$ to the affine Weyl group of $G$, which is the group generated by reflections in the hyperplanes determined by the set of affine roots $\{\alpha+r \mid \alpha \in \Phi, r \in Z\}$. Let $Y_{r}=U_{-\alpha-r} / U_{-\alpha-r+1}$. Then

$$
\begin{aligned}
\int_{U_{\alpha}} \chi_{\mathrm{Re} \lambda}\left(u \bar{w}_{\alpha}\right) d u & =\int_{U_{-\alpha}} \chi_{\mathrm{Re} \lambda}\left(\bar{w}_{\alpha} v\right) d v \\
& =\int_{U_{-\alpha}} \chi_{\mathrm{Re} \lambda}(v) d v \\
& =\int_{U_{-\alpha+0}} d v+\sum_{r=1}^{\infty} \int_{Y_{r}} \chi_{\mathrm{Re} \lambda}(v) d v .
\end{aligned}
$$

We may write $v \in U_{-\alpha-r}$ as $v=u_{1} n u_{2}$, where $u_{1}, u_{2} \in U_{\alpha+r} \subset U \cap K$ ( $r$ is a positive interger) and $\nu(n)=w_{\alpha-r}$. If $n_{\alpha} \in K$ with $\nu\left(n_{\alpha}\right)=w_{\alpha}$, then $n_{\alpha} n \in T$ and $\nu\left(n_{\alpha} n\right)=t_{\alpha}^{r}$ where $t_{\alpha}$ is the translation $x \mapsto x+\alpha^{\nu}$ in the affine Weyl group. Let $q_{\alpha}=\left(U_{\alpha-1}: U_{\alpha}\right)$ and $q_{\alpha / 2}=q_{\alpha+1} q_{\alpha}^{-1}$.

Thus

$$
\begin{aligned}
\int_{Y r} \chi_{\mathrm{Re \lambda} \lambda}(v) & =\int_{Y_{r}} \chi_{\mathrm{Re} \lambda}\left(u_{1} n u_{2}\right)=\int_{Y_{r}} \chi_{\mathrm{Re} \lambda}(n)=\int_{Y_{r}} \chi_{\mathrm{Re \lambda} \lambda}\left(n_{\alpha} n\right) \\
& =\operatorname{Re} \lambda^{-1} \rho^{-1}\left(t_{\alpha}^{r}\right) \cdot \operatorname{vol}\left(Y_{r}\right) \\
& =\operatorname{Re} \lambda^{-1}\left(t_{\alpha}\right)^{r} q_{\alpha / 2}^{-r / 2} q_{\alpha}^{-r}\left[q_{\alpha / 2}^{[r / 2]} q_{\alpha}^{r}-q_{\alpha / 2}^{[r-1 / 2]} q_{\alpha}^{r-1}\right] .
\end{aligned}
$$

Thus the sum over $r$ is a geometric series with common ratio $\operatorname{Re} \lambda\left(t_{\alpha}\right)^{-2}$, which converges if and only if $s=\operatorname{Re} \lambda_{\alpha}>0$.

The value of the sum is then given by Harish-Chandra's $c$-function $c_{0}(\alpha, s)=c(\alpha / 2, s) c(\alpha, s)$. The reader is referred to Macdonald [27].

Let $V$ be the unipotent radical of the Borel opposed to $B$. Since $G=V B$ up to a set of Haar measure zero, functions in $H_{2}$ are determined by their values on $V$ and we may realize $\operatorname{Ind}_{B}^{G} \lambda$ on $L^{2}(V)$. Assume that $\operatorname{Re} \lambda_{\alpha}>0$. If $G$ is a Chevalley group, then $U_{-\alpha}$ is onedimensional, and a calculation realizing the representation on $L^{2}(V)$ via the Fourier transform in $U_{-\alpha}$, as in the $\chi$-realization of Gelfand, Graev and Pyatetskii-Shapiro [7] or Sally [28] for SL (2), shows that $A\left(\bar{w}_{\alpha}, \lambda\right)$ acts as multiplication by $\lambda_{\alpha}^{-1} \Gamma\left(\lambda_{\alpha}\right)$, where $\bar{w}_{\alpha}=w_{\alpha}(1)$. We may then use the analytic continuation of the gamma function to define the intertwining operator $A\left(w_{\alpha}, \lambda\right)$ for any quasi-character $\lambda$ such that $\Gamma\left(\lambda_{\alpha}\right)$ is defined, i.e., for $\lambda_{\alpha} \neq \equiv 1$. 
If we normalize $A\left(\bar{w}_{\alpha}, \lambda\right)$ by $\Gamma\left(\lambda_{\alpha}\right)$ by setting $a\left(\bar{w}_{\alpha}, \lambda\right)=$ $\left(1 / \Gamma\left(\lambda_{\alpha}\right)\right) A\left(\bar{w}_{\alpha}, \lambda\right)$, then by analytic continuation $a\left(\bar{w}_{\alpha}, \lambda\right)$ defines an intertwining operator between $\operatorname{Ind}_{B}^{G} \lambda$ and $\operatorname{Ind}_{B}^{G} w_{\alpha} \lambda$ for all $\lambda$.

Suppose $w \in W$ has length $l$ and $w=w_{\alpha_{1}} \cdots w_{\alpha_{l}}$ is a reduced product of basic reflections, $\alpha_{i}$ simple. The appropriate normalizing factor for $A(\bar{w}, \lambda)=A\left(\bar{w}_{\alpha_{1}}, w_{\alpha_{2}} \cdots w_{\alpha_{l}} \lambda\right) \circ \cdots \circ A\left(\bar{w}_{\alpha l}, \lambda\right)$ is

$$
\prod_{i=1}^{l} \Gamma\left(w_{\alpha_{i+1}} \cdots w_{\alpha_{l}} \lambda_{\alpha_{i}}\right)=\prod_{\alpha \in R(w)} \Gamma\left(\lambda_{\alpha}\right) .
$$

Denote this product by $\Gamma_{w}(\lambda)$ and define

$$
\mathfrak{a}(\bar{w}, \lambda)=\frac{1}{\Gamma_{w}(\lambda)} A(\bar{w}, \lambda) .
$$

An argument similar to that in Winarsky [37] gives the analytic continuation of $A(\bar{w}, \lambda)$ and $a(\bar{w}, \lambda)$ in the case of a semi-simple $p$-adic algebraic group.

THEOREM 1. Let $G$ be a connected semi-simple p-adic group and suppose $f \in H_{\lambda}$ is locally constant. The map $\lambda \mapsto(A(\bar{w}, \lambda) f)(k)$ of $D(w)$ into $C$ is analytic for $k \in K$. It extends to $C^{n}$ as a meromorphic function. When $\lambda$ is not a pole of the extension, the operators $A(\bar{w}, \lambda)$ intertwine the representations $\operatorname{Ind}_{B}^{G} \lambda$ and $\operatorname{Ind}_{B}^{G} w \lambda$.

Proof. The unramified part of $\lambda$ is determined by $n$ unramified characters $|\cdot|^{s_{\alpha}}, \alpha$ simple, each of which is identified with the complex number $s_{\alpha}$. Multiply this by a representation $\lambda^{*}$ of ker $\nu$. Considering $\lambda^{*}$ fixed and letting the unramified part of $\lambda$ vary, we identify $\lambda$ with a point in $\boldsymbol{C}^{n}$.

It is enough to prove the theorem in the case $w=w_{\alpha}$ is a simple reflection. Again, we follow Macdonald [27]. Choose a coset representative $n_{\alpha} \in K$ for $w_{\alpha}$ with $\nu\left(n_{\alpha}\right)=w_{\alpha}$. Write $v \in Y_{r}=U_{-\alpha-r} \mid U_{-\alpha-r+1}$ as $v=u_{1} n_{\alpha}^{-1} t_{\alpha}^{r} u_{2}$, with $u_{1}, u_{2} \in U_{\alpha+r}$ and $\nu\left(t_{\alpha}\right)$ translation by $\alpha^{\nu}$. Suppose that $f$ is constant on cosets of $U_{-\alpha+m}$ in $K$.

Then

$$
\begin{aligned}
A\left(n_{\alpha}, \lambda\right) f(k) & =\int_{U_{-\alpha}} f\left(k n_{\alpha} v\right) d v \\
& =\int_{U_{-\alpha+m-1}} f\left(k n_{\alpha} v\right) d v+\sum_{r=m}^{\infty} \int_{Y_{r}} f\left(k n_{\alpha} u_{1} n_{\alpha}^{-1} t_{\alpha}^{r} u_{2}\right) \\
& =\int_{U_{-\alpha+m-1}}^{\infty} f\left(k n_{\alpha} v\right) d v+\sum_{r=m}^{\infty} \int_{Y_{r}} f\left(k n_{\alpha} u_{1} n_{\alpha}^{-1}\right) \lambda^{-1} \rho^{-1}\left(t_{\alpha}^{r}\right) .
\end{aligned}
$$

But $n_{\alpha} u_{1} n_{\alpha}^{-1} \in U_{-\alpha+m}$ and $f$ is assumed constant on this. The sum over $r$ is thus 


$$
\begin{aligned}
& f(k) \sum_{r=m}^{\infty} \int_{Y_{r}} \lambda^{-1} \rho^{-1}\left(t_{\alpha}^{r}\right) \\
& = \begin{cases}0 & \text { if } \lambda_{\alpha} \text { is ramified } \\
f(k) \sum_{r=m}^{\infty} \lambda\left(t_{\alpha}\right)^{-r} q_{\alpha / 2}^{-r / 2} q_{\alpha}^{-r}\left(q_{\alpha / 2}^{[r / 2]} q_{\alpha}^{r}-q_{\alpha / 2}^{[[r-1) ! 2]} q_{\alpha}^{r-1}\right) & \text { if } \lambda_{\alpha} \text { is unramified . }\end{cases}
\end{aligned}
$$

For $\lambda_{\alpha}$ unramified, this is a geometric series with common ratio $\lambda\left(t_{\alpha}\right)^{-2}$, which converges if and only in $\operatorname{Re} \lambda_{\alpha}>0$. In this case the sum is given by

$$
f(k) \cdot \frac{\left(1-q_{\alpha}^{-1}\right)\left(1+\lambda\left(t_{\alpha}\right)^{-1} q_{\alpha / 2}^{-1 / 2}\right) \lambda\left(t_{\alpha}\right)^{-m}}{1-\lambda\left(t_{\alpha}\right)^{-2}}
$$

We note that if $G$ is split, then $q_{\alpha}=q$ and $q_{\alpha / 2}=1$ and the above sum agrees with Winarsky's.

Thus $\lambda \mapsto A\left(n_{\alpha}, \lambda\right) f(k)$ extends to a meromorphic function of $s_{\alpha}$ with simple poles at $\lambda\left(t_{\alpha}\right)= \pm 1$ for $q_{\alpha / 2} \neq 1$ and at $\lambda\left(t_{\alpha}\right)=1$ for $q_{\alpha / 2}=1$, if $|\cdot|^{s} \alpha$ is unramified, and extends to an analytic function if $\lambda_{\alpha}$ is ramified. By analytic continuation, the intertwining relation holds if $\lambda$ is not a pole of the extension.

If we normalize $A\left(\bar{w}_{\alpha}, \lambda\right)$ by Harish-Chandra's $c$-function $c_{0}\left(\alpha, \lambda_{\alpha}\right)$ and $A(\bar{w}, \lambda)$ by $c_{w}(\lambda)=\prod_{\alpha \in R(w)} c_{0}\left(\alpha, \lambda_{\alpha}\right)$ then $\lambda \mapsto \mathfrak{a}(\bar{w}, \lambda)=\left(1 / c_{w}(\lambda)\right) A(\bar{w}, \lambda)$ extends to a meromorphic function on $\boldsymbol{C}^{n}$ which is holomorphic in a neighborhood of $\left\{\left(c_{1}, \cdots, c_{n}\right) \in C^{n} \mid \operatorname{Re} c_{i}=0, i=1, \cdots, n\right\}$ and defines an intertwining operator between $\operatorname{Ind}_{B}^{G} \lambda$ and $\operatorname{Ind}_{B}^{G} w \lambda$ if $\lambda$ is not a pole.

An argument similar to that of [37] shows that $\operatorname{Ind}_{B}^{G} \lambda$ is reducible if there exists a $w \in W, w \neq 1$ with $w \lambda=\lambda$ such that $\lambda$ is not a pole of $c_{w}(\lambda)$.

2. The cocycle condition for $a(w, \lambda)$. We now choose certain coset representatives for each $w \in W$. Fix any coset representatives $n_{\alpha}$ for the basic reflections $w_{\alpha}, \alpha$ simple. Suppose $w \in W$ has length $l$ and $w=w_{\alpha_{1}} w_{\alpha_{2}} \cdots w_{\alpha_{l}}$ is a reduced product of basic reflections. We take $n_{\alpha_{1}} n_{\alpha_{2}} \cdots n_{\alpha_{l}}$ as the coset representative of $w$ and define

$$
\begin{aligned}
A(w, \lambda) & =A\left(n_{\alpha_{1}} n_{\alpha_{2}} \cdots n_{\alpha_{l}}, \lambda\right) \text { and } \\
\mathfrak{a}(w, \lambda) & =\mathfrak{a}\left(n_{\alpha_{1}} n_{\alpha_{2}} \cdots n_{\alpha_{l}}, \lambda\right) .
\end{aligned}
$$

This is well-defined by the following.

THEOREM 1. Fix coset representatives $n_{\alpha} \in N(T)$ for the basic reflections $w_{\alpha}$, $\alpha$ simple. Suppose $w$ is expressed as a reduced product $w_{\alpha_{1}} w_{\alpha_{2}} \cdots w_{\alpha_{l}}$ of basic reflections, $l(w)=l$. Then the coset 
representative $n_{\alpha_{1}} n_{\alpha_{2}} \cdots n_{\alpha_{l}}$ of $w$ is independent of the expression $w_{\alpha_{1}} w_{\alpha_{2}} \cdots w_{\alpha_{l}}$.

Proof. For Chevalley groups, see page 242 of [34]. For connected reductive $p$-adic groups, see page 112 of [4].

We now fix a set of coset representatives as above and write $A(w, \lambda)$ instead of $A(\bar{w}, \lambda)$. For the calculations in Chapter 3, we have taken $n_{\alpha}=w_{\alpha}(1)$ as the coset representative for the basic reflection $w_{\alpha}, \alpha$ simple.

Recall the cocycle condition $A\left(w^{\prime} w^{\prime \prime}, \lambda\right)=A\left(w^{\prime}, w^{\prime \prime} \lambda\right) \circ A\left(w^{\prime \prime}, \lambda\right)$ if $l\left(w^{\prime} w^{\prime \prime}\right)=l\left(w^{\prime}\right)+l\left(w^{\prime \prime}\right)$. In this case we also have $\Gamma_{w^{\prime} w^{\prime \prime}}(\lambda)=$ $\Gamma_{w^{\prime}}\left(w^{\prime \prime} \lambda\right) \Gamma_{w^{\prime \prime}}(\lambda)$ since $R\left(w^{\prime} w^{\prime \prime}\right)=R\left(w^{\prime \prime}\right) \cup w^{\prime \prime-1} R\left(w^{\prime}\right)$. Thus $a\left(w^{\prime} w^{\prime \prime}, \lambda\right)=$ $\mathfrak{a}\left(w^{\prime}, w^{\prime \prime} \lambda\right) \circ \mathfrak{a}\left(w^{\prime \prime}, \lambda\right)$ if $l\left(w^{\prime} w^{\prime \prime}\right)=l\left(w^{\prime}\right)+l\left(w^{\prime \prime}\right)$.

We will show that with the above choice of coset representatives, the cocycle condition holds for the normalized operators $a(w, \lambda)$ with no condition on the lengths of $w^{\prime}$ and $w^{\prime \prime}$.

We have seen that under the $\chi$-realization in $U_{-\alpha}, A\left(w_{\alpha}, \lambda\right)$ acts as multiplication by $\lambda_{\alpha}^{-1} \Gamma\left(\lambda_{\alpha}\right)$. Thus $A\left(w_{\alpha}, w_{\alpha} \lambda\right) \circ A\left(w_{\alpha}, \lambda\right)=\Gamma\left(\lambda_{\alpha}\right) \Gamma\left(\lambda_{\alpha}^{-1}\right)$ is scalar and $a\left(w_{\alpha}, w_{\alpha} \lambda\right) \circ \mathfrak{a}\left(w_{\alpha}, \lambda\right)=I$ is the identity.

Thus $\mathfrak{a}\left(w_{\alpha}, w_{\alpha} \lambda\right)$ is the inverse of $\mathfrak{a}\left(w_{\alpha}, \lambda\right)$, i.e., the cocycle condition holds in this case.

THEOREM 2. The cocycle condition $\mathfrak{a}\left(w^{\prime} w^{\prime \prime}, \lambda\right)=\mathfrak{a}\left(w^{\prime}, w^{\prime \prime} \lambda\right) \circ \mathfrak{a}\left(w^{\prime \prime}, \lambda\right)$ holds with no condition on the lengths of $w^{\prime}$ and $w^{\prime \prime}$.

Proof. We first recall that with our choice of coset representatives the operators are well-defined. This is in fact equivalent to the cocycle condition.

The proof is by induction on the length of $w^{\prime}$. Suppose $l\left(w^{\prime}\right)=1$, say $w^{\prime}=w_{\alpha}, \alpha$ simple. If $l\left(w_{\alpha} w^{\prime \prime}\right)=l\left(w^{\prime \prime}\right)+1$, then we are done. Otherwise $l\left(w_{\alpha} w^{\prime \prime}\right)=l\left(w^{\prime \prime}\right)-1$. Suppose $w^{\prime \prime}=w_{\beta_{1}} w_{\beta_{2}} \cdots w_{\beta_{l}}$ is a reduced expression for $w^{\prime \prime}$ as a product of simple reflections. Then by Coxeter's exchange condition [2], $w^{\prime} w^{\prime \prime}=w_{\alpha} w_{\beta_{1}} w_{\beta_{2}} \cdots w_{\beta_{l}}=$ $w_{\beta_{1}} \cdots \widehat{w}_{\beta_{j}} \cdots w_{\beta_{l}}$, where $\beta_{j}$ is omitted.

Since $w_{\alpha}$ has order $2, w^{\prime \prime}=w_{\beta_{1}} \cdots w_{\beta_{l}}=w_{\alpha} w_{\beta_{1}} \cdots \hat{w}_{\beta_{j}} \cdots w_{\beta_{l}}$, and these are both reduced expressions for $w^{\prime \prime}$. Then since $a\left(w^{\prime \prime}, \lambda\right)$ does not depend on the reduced expression chosen for $w^{\prime \prime}$, we get

$$
\begin{aligned}
\mathfrak{a}\left(w_{\alpha},\right. & \left.w^{\prime \prime} \lambda\right) \circ \mathfrak{a}\left(w^{\prime \prime}, \lambda\right) \\
& =\mathfrak{a}\left(w_{\alpha}, w^{\prime \prime} \lambda\right) \circ \mathfrak{a}\left(w_{\alpha} w_{\beta_{1}} \cdots \hat{w}_{\beta_{i}} \cdots w_{\beta_{l}}, \lambda\right) \\
& =\mathfrak{a}\left(w_{\alpha}, w^{\prime \prime} \lambda\right) \circ \mathfrak{a}\left(w_{\alpha}, w_{\beta_{1}} \cdots \hat{w}_{\beta_{j}} \cdots w_{\beta_{l}} \lambda\right) \circ \mathfrak{a}\left(w_{\beta_{1}} \cdots \hat{w}_{\beta_{j}} \cdots w_{\beta_{l}}, \lambda\right) \\
& =I \circ \mathfrak{a}\left(w_{\beta_{1}} \cdots \hat{w}_{\beta_{j}} \cdots w_{\beta_{l}}, \lambda\right) \\
& =a\left(w_{\alpha} w^{\prime \prime}, \lambda\right),
\end{aligned}
$$


since $l\left(w_{\alpha} w_{\beta_{1}} \cdots \hat{w}_{\beta_{j}} \cdots w_{\beta_{l}}\right)=1+l\left(w_{\beta_{1}} \cdots \hat{w}_{\beta_{i}} \cdots w_{\beta_{l}}\right)$ and $w_{\beta_{1}} \cdots$ $\hat{w}_{\beta_{j}} \cdots w_{\beta_{l}}$ is a reduced expression for $w_{\alpha} w^{\prime \prime}$.

Thus the theorem is true if $w^{\prime}$ has length 1 . Suppose $w^{\prime}$ has length $>1$ and write $w^{\prime}=w_{\alpha} w_{1}$ with $\alpha$ simple and $l\left(w_{1}\right)=l\left(w^{\prime}\right)-1$. Then

$$
\begin{aligned}
\mathfrak{a}\left(w^{\prime} w^{\prime \prime}, \lambda\right)= & \mathfrak{a}\left(w_{\alpha} w_{1} w^{\prime \prime}, \lambda\right)=\mathfrak{a}\left(w_{\alpha}, w_{1} w^{\prime \prime} \lambda\right) \mathfrak{a}\left(w_{1} w^{\prime \prime}, \lambda\right) \\
= & \mathfrak{a}\left(w_{\alpha}, w_{1} w^{\prime \prime} \lambda\right) \mathfrak{a}\left(w_{1}, w^{\prime \prime} \lambda\right) \mathfrak{a}\left(w^{\prime \prime}, \lambda\right) \\
& \quad \text { by the induction hypothesis, } \\
= & \mathfrak{a}\left(w_{\alpha} w_{1}, w^{\prime \prime} \lambda\right) \mathfrak{a}\left(w^{\prime \prime}, \lambda\right) \text { since } l\left(w_{\alpha}\right)=1, \\
= & a\left(w^{\prime}, w^{\prime \prime} \lambda\right) \mathfrak{a}\left(w^{\prime \prime}, \lambda\right) .
\end{aligned}
$$

Thus the cocycle condition is true with no condition on the lengths of $w^{\prime}$ and $w^{\prime \prime}$. We remark that one could also use the relations $\left(w_{\alpha} w_{\beta}\right)^{n(\alpha, \beta)}=1$ defining $W$ as a Coxeter group to prove the cocycle condition.

We note that to prove the theorem, we need only normalize the operators so that $\mathfrak{a}\left(w_{\alpha}, w_{\alpha} \lambda\right)$ is the inverse of $\mathfrak{a}\left(w_{\alpha}, \lambda\right)$. For Chevalley groups we may do this with either gamma functions or $c$-functions.

For Macdonald's "groups of $p$-adic type" we may use the $c$-functions to do this, at least for unramified $\lambda$. In any case, $\mathfrak{a}\left(w^{-1}, w \lambda\right) \mathfrak{a}(w, \lambda)$ is scalar. If $\lambda$ is unramified and $f_{\lambda}$ is the $K$-fixed vector in $H_{\lambda}$ with $f_{\lambda}(e)=1$, then $A(w, \lambda) f_{\lambda}=c_{w}(\lambda) f_{w \lambda}$ and $A\left(w^{-1}, w \lambda\right) A(w, \lambda) f_{\lambda}=$ $c_{w^{-1}}(w \lambda) c_{w}(\lambda) f_{\lambda}$. So if $\mathfrak{a}(w, \lambda)=\left(1 / c_{w}(\lambda)\right) A(w, \lambda)$, we see that $\mathfrak{a}\left(w^{-1}, w \lambda\right) \mathfrak{a}(w, \lambda)=I$.

Thus the cocycle relation holds with no condition on lengths for "groups of $p$-adic type" and unramified characters $\lambda$.

Finally, we note that the cocycle condition implies that $w \mapsto a(w, \lambda)$ is a representation of $W_{\lambda}=\{w \in W \mid w \lambda=\lambda\}$.

3. The Knapp-Stein $R$-group. * We define a subgroup $R$ of $W_{\lambda}$ such that the commuting algebra of $\operatorname{Ind}_{B}^{G} \lambda$ is given as the group algebra $C[R]$. The theory of the $R$-group was developed by Knapp and Stein for real semi-simple Lie groups. The following $p$-adic analogue is another illustration of Harish-Chandra's "Lefschetz principle," which says that whatever is true for real reductive groups is also true for $p$-adic groups.

Let $\Delta^{\prime}=\left\{\alpha>0 \mid \lambda_{\alpha} \equiv 1\right\}$. Then $\pm \Delta^{\prime}$ is a sub-root system of the root system $\Phi$.

Let

$$
\begin{aligned}
R & =\left\{w \in W_{\lambda} \mid \alpha>0 \text { and } \lambda_{\alpha} \equiv 1 \text { imply that } w \alpha>0\right\} \\
& =\left\{w \in W_{\lambda} \mid w\left(\Delta^{\prime}\right)=\Delta^{\prime}\right\} .
\end{aligned}
$$

* Suppose that $G$ is a Chevalley group. 
Let $W^{\prime}$ be the reflection group associated to $\pm \Delta^{\prime}$, i.e., the group generated by the reflections $\left\{w_{\alpha} \mid \alpha \in \Delta^{\prime}\right\}$.

THEOREM 1. $W_{\lambda}$ can be written as a semi-direct product $W_{\lambda}=$ $R \ltimes W^{\prime}$, where $R$ and $W^{\prime}$ are defined above. Further, $W^{\prime}$ is the group $\left\{w \in W_{\lambda} \mid a(w, \lambda)\right.$ is scalar $\}$.

Proof. First we show that $W^{\prime} \leqq W_{\lambda}$. Let $\alpha \in \Delta^{\prime}$ and show that $w_{\alpha} \lambda_{\beta}=\lambda_{\beta}$ for all roots $\beta$. But since $\lambda_{\alpha} \equiv 1, \quad w_{\alpha} \lambda_{\beta}=\lambda_{w_{\alpha} \beta}^{-1}=$ $\lambda_{\beta} \lambda_{\alpha}^{-\left\langle\alpha^{v}, \beta^{v}\right\rangle}=\lambda_{\beta}$.

Now suppose $w \in W_{\lambda}$ has length $l$ and write $w=w_{\alpha_{1}} \cdots w_{\alpha_{l}}$ as a reduced product of basic reflections. If $w \in R$ then we are done. Otherwise there exists $\alpha \in \Delta^{\prime}$ with $w \alpha<0$. Then $\alpha=w_{\alpha_{l}} \cdots w_{\alpha_{i+1}}\left(\alpha_{i}\right)$ for some $i, 1 \leqq i \leqq l$. Let $r=w_{\alpha_{1}} \cdots \hat{w}_{\alpha_{i}} \cdots w_{\alpha_{l}}$ where $w_{\alpha_{i}}$ is omitted. Then

$$
\begin{aligned}
w & =w_{\alpha_{1}} \cdots \hat{w}_{\alpha_{i}} \cdots w_{\alpha_{l}} w_{\alpha_{l}} \cdots \hat{w}_{\alpha_{i}} \cdots w_{\alpha_{1}} w_{\alpha_{1}} \cdots w_{\alpha_{l}} \\
& =r w_{\alpha_{l}} \cdots w_{\alpha_{i+1}} w_{\alpha_{i}} w_{\alpha_{i+1}} \cdots w_{\alpha_{l}} \\
& =r w_{w_{\alpha l} \cdots w_{\alpha_{i+1}}(\alpha i)} \\
& =r w_{\alpha} .
\end{aligned}
$$

Then $w_{\alpha} \in W^{\prime}$ since $\alpha \in \Delta^{\prime}$. Since $l(r)<l(w)$ we may use induction on $l(w)$ to complete the proof that $W_{\lambda}=R \times W^{\prime}$.

Finally, we show that $W^{\prime}=\left\{w \in W_{\lambda} \mid \mathfrak{a}(w, \lambda)\right.$ is scalar $\}$. In the $\chi$-realization, $\mathrm{a}\left(w_{\alpha}, \lambda\right)$ acts as multiplication by $\lambda_{\alpha}^{-1}$, if we use $w_{\alpha}(1)$ as coset representative for $w_{\alpha}$ and normalize the operator by the gamma function. Thus $\mathfrak{a}\left(w_{\alpha}, \lambda\right)=I$ if and only if $\alpha \in \Delta^{\prime}$. Then $\mathfrak{a}(w, \lambda)=I$ for all $w \in W^{\prime}$. The cocycle condition shows that $w \rightarrow$ $\mathrm{a}(w, \lambda)$ is a homomorphism from $W_{\lambda}$ into the group of invertible intertwining operators for $\operatorname{Ind}_{B}^{G} \lambda$, and Winarsky [37] shows that $a(w, \lambda)$ is nonscalar if $w \in R, w \neq 1$. These observations complete the proof of the theorem.

We note that Winarsky's condition for reducibility is essentially that $R$ is nontrivial.

By an unpublished theorem of Harish-Chandra, the commuting algebra $C(\lambda)$ of $\operatorname{Ind}_{B}^{G} \lambda$ is spanned by $\left\{\mathfrak{a}(w, \lambda) \mid w \in W_{\lambda}\right\}$. By the above, it is spanned by $\{a(w, \lambda) \mid w \in R\}$. But these operators are linearly independent, by our calculations in Chapter 3, or by an appeal to Silberger's theorem [33], which states that

$$
\operatorname{dim} C(\lambda)=\left|W_{\lambda}\right| /\left|W^{\prime}\right| \text {. }
$$

Thus the operators $\{\mathfrak{a}(w, \lambda) \mid w \in R\}$ form a basis for $C(\lambda)$. Finally, since $\mathfrak{a}\left(w^{\prime} w^{\prime \prime}, \lambda\right)=\mathfrak{a}\left(w^{\prime}, \lambda\right) \mathfrak{a}\left(w^{\prime \prime}, \lambda\right)$ for $w^{\prime}$ and $w^{\prime \prime}$ in $R \leqq W_{\lambda}$, we have the following 
THEOREM 2. The commuting algebra $C(\lambda)$ of the (unitary) principal series representation $\operatorname{Ind}_{B}^{G} \lambda$ is isomorphic to the group algebra $C[R]$.

COROLLARY 1.

(a) $\operatorname{dim} C(\lambda)=|R|$.

(b) The number of inequivalent irreducible components of $\operatorname{Ind}_{B}^{G} \lambda$ is equal to the dimension of the center of $C[R]$, which equals the number of conjugacy classes in $R$.

(c) $\operatorname{Ind}_{B}^{G} \lambda$ decomposes with multiplicities equal to 1 if and only if $R$ is abelian.

(d) If $\boldsymbol{C}[R]=M_{n_{1}}(\boldsymbol{C}) \oplus \cdots \oplus M_{n_{k}}(\boldsymbol{C})$, then $n_{1}, \cdots, n_{k}$ are the multiplicities of the irreducible components of $\operatorname{Ind}_{B}^{G} \lambda$.

\section{CHAPTER II \\ CLASSIFICATION OF THE $R$-GROUPS}

The $R$-groups which occur for Chevalley groups of each type $\mathrm{A}_{n}, \mathrm{~B}_{n}, \mathrm{C}_{n}, \mathrm{D}_{n}, \mathrm{E}_{6}, \mathrm{E}_{7}, \mathrm{E}_{8}, \mathrm{~F}_{4}$ and $\mathrm{G}_{2}$, are determined. They are abelian except in the cases of $\mathrm{D}_{n}$, for which non-abelian $R$ occur for every $n \geqq 4$, and in the cases $\mathrm{E}_{7}$ and $\mathrm{E}_{8}$.

The orders of the $R$-groups which can occur depend on $n$ and on the arithmetic of the field $\mathfrak{k}$. Further, the existence of the nonabelian $\mathrm{E}_{8} R$-group depends on the arithmetic of $\mathfrak{t}$.

Let $\lambda$ be a character of $T$ and let

$$
\begin{aligned}
\Delta^{\prime} & =\left\{\alpha>0 \mid \lambda_{\alpha} \equiv 1\right\} \\
& =\left\{\alpha>0 \mid a\left(w_{\alpha}, \lambda\right) \text { is scalar }\right\} .
\end{aligned}
$$

Then

$$
\begin{aligned}
R & =\left\{w \in W_{\lambda} \mid \alpha>0 \text { and } \lambda_{\alpha} \equiv 1 \text { imply that } w \alpha>0\right\} \\
& =\left\{w \in W_{\lambda} \mid w\left(\Delta^{\prime}\right)=\Delta^{\prime}\right\} .
\end{aligned}
$$

We note that the second definition of $R$ shows that it is a group.

Identify $\lambda_{\alpha}$ with $\alpha^{v}$ in the root system $\Phi^{v}$ dual to $\Phi$ and let $\mathscr{L}=\mathscr{L}_{\lambda}=\boldsymbol{Z}\left[\sum_{\alpha} m_{\alpha} \cdot \alpha^{v} \mid \Pi_{\alpha} \lambda_{\alpha}^{m_{\alpha}}=1, m_{\alpha} \in Z\right]$. Then $w \in W_{\lambda}$ if and only if $\alpha^{v}-w \alpha^{v} \in \mathscr{L}$ for all simple roots $\alpha^{v}$. $\mathscr{L}$ contains the set $\left\{\alpha^{v} \mid \alpha \in \Delta^{\prime}\right\}=$ positive elements in $\mathscr{L} \cap \Phi^{v}$, which we sometimes denote by $\Delta^{\prime}$.

$w$ acts on $\lambda_{\alpha}$ as $w$ acts on $\alpha^{v}$, as $w^{-1}$ acts on $\alpha$. Since $w \in R$ if and only if $w^{-1} \in R$,

$R=\left\{w \in W_{\lambda} \mid \alpha^{v} \in \Phi^{v}, \alpha^{v} \in \mathscr{L}\right.$ and $\alpha^{v}>0$ imply that $\left.w \alpha^{v}>0\right\}$.

We do the calculations to classify $R$ in the root system $\Phi^{v}$ dual 
to $\Phi$. Note that not all of $w^{i}\left(\alpha^{v}-w \alpha^{v}\right), 0 \leqq i<$ ord $w$, can be positive, since their sum is zero. Thus, if $\alpha^{v}-w \alpha^{v} \in \mathscr{L}_{\lambda} \cap \Phi_{+}^{v}$ for some root $\alpha$, then $w \notin R_{\lambda}$. Note that this condition is invariant under conjugation, replacing $\lambda$ by $w \lambda$, although $R_{w \lambda}$ may not be equal to ${ }^{w} R_{\lambda}=w R_{\lambda} w^{-1}$.

We use this observation to determine which elements of $W$ can form an $R$-group for some $\lambda \in T^{\wedge}$. Once we have a possible $R$, we look for a character $\lambda$ with $R \leqq W_{\lambda}$ as $R$-group. The existence of such a $\lambda$ depends on the arithmetic of $\mathfrak{f}$. Our proof explicitly constructs a list of $\lambda$ and $R$ and shows that any nontrivial $R$-group is conjugate under $W$ to one on the list.

We proceed according to the classification of types of root systems [2].

1. Type $\mathrm{A}_{n} . \quad \Phi=\Phi^{v}=\left\{e_{i}-e_{j} \mid 1 \leqq i \neq j \leqq n+1\right\}$ is self-dual and the Weyl group $W \cong S_{n+1}$ acts as permutations of the $e_{i}$.

THEOREM $\mathrm{A}_{n} . \quad R$ is abelian and $|R|$ divides $n+1$. If the largest cyclic subgroup of $R$ has order $m$, then $|R|$ divides $\left[\mathfrak{k}^{*}:\left(\mathfrak{k}^{*}\right)^{m}\right]=$ order of the subgroup of $\left(\mathfrak{f}^{*}\right)^{\wedge}$ consisting of characters of order dividing $\mathrm{m}$.

Conventions. We identify $e_{i}-e_{j} \in \Phi^{v}$ with the character $\lambda_{e_{i-e_{j}}}$ and consider $Z\left[\Phi^{v}\right] / \mathscr{L}$ as a subgroup of $\left(\mathfrak{t}^{*}\right)^{\wedge}$ by the map $\sum m_{\alpha} \alpha^{v} \mapsto$ II $\lambda_{\alpha}^{m_{\alpha}}$.

LEMMA 1. $w \mapsto e_{i}-e_{w i}$ is an injective homomorphism from $R$ into $\left(\mathfrak{f}^{*}\right)^{\wedge}$, independent of $i$.

Proof. Let $w \in W_{\lambda}$. Then $e_{i}-e_{j}=w\left(e_{i}-e_{j}\right)=e_{w i}-e_{w j}$ implies that $e_{i}-e_{w i}=e_{j}-e_{w j}$, so that the map is independent of $i$. Note that $=$ means congruence $\bmod \mathscr{L}$ and that we have used the fact that $w \in W_{2}$ if and only if $\alpha^{v}-w \alpha^{v} \in \mathscr{L}$ for all $\alpha^{v} \in \Phi^{v}$.

Let $w, w^{\prime} \in W_{\lambda}$. Then $e_{i}-e_{w w^{\prime} i}=e_{i}-e_{w^{\prime} i}+e_{w^{\prime} i}-e_{w\left(w^{\prime} i\right)}=e_{i}-$ $e_{w^{\prime} i}+e_{i}-e_{w i}$ shows that the map is a homomorphism.

If $w \neq 1$ then we may replace everything by a conjugate to assume that $w 1 \neq 1$. Then if $e_{1}-e_{w 1} \in \mathscr{L}$, we have $e_{1}-e_{w 1} \in \Delta^{\prime}$ and $w^{-1}\left(e_{1}-e_{w 1}\right)<0$, so that $w \notin R$. Thus the map is injective on $R$.

Thus $R$ is isomorphic to a subgroup of $\left(\mathfrak{l}^{*}\right)^{\wedge}$ and is abelian. Further, if the largest cyclic subgroup of $R$ has order $m$, then any element of $R$ has order dividing $m$ and the image of $R$ is contained in the subgroup of characters of $\mathfrak{1}^{*}$ of order dividing $m$. Thus $|R|$ divides $\left[\mathfrak{f}^{*}:\left(\mathfrak{l}^{*}\right)^{m}\right]$.

Since $R$ is abelian, $\operatorname{Ind}_{B}^{G} \lambda$ decomposes simply. This is shown for $G=\mathrm{SL}(n, \mathfrak{f})$ by Howe and Silberger [12]. 
We note that if $=\boldsymbol{R}$, then the image of $R$ is a finite subgroup of $\left(\boldsymbol{R}^{*}\right)^{\wedge}$, so has order 1 or $2[17]$.

Lemma 2. The stabilizer of any $e_{i}$ in $R$ is trivial. Thus $|R|$ divides $n+1$.

Proof. Suppose $w \in R$ fixes some $i$. Then $e_{i}-e_{w i}=0$ and the image of $w$ under the above map is trivial. Thus $w=1$. So the action of $R$ partitions $\{1,2, \cdots, n+1\}$ into orbits of cardinality $|R|$ and $|R|$ divides $n+1$.

Note that any finite subgroup of $\left(\mathfrak{k}^{*}\right)^{\wedge}$ with order dividing $n+1$ is the image of some $R$-group.

REMARK. The homomorphism $w \mapsto e_{i}-e_{w i}$ is suggested by the following. In Chapter 3 we realize $\operatorname{Ind}_{B}^{G} \lambda$ and $\mathfrak{a}(w, \lambda)$ on $L^{2}(V)$. We exhibit a class of functions in $L^{2}(V)$ on which $a\left(w_{\alpha}, \lambda\right)$ acts as multiplication by $M\left(w_{\alpha}, \lambda\right)=\lambda_{\alpha}^{-1}$ in the $U_{-\alpha}$ coordinate, $\alpha$ simple. Then $\mathfrak{a}(w, \lambda)=\mathfrak{a}\left(w_{\alpha_{1}} \cdots w_{\alpha_{l}}, \lambda\right)$ acts as multiplication by the function $M(w, \lambda)=M\left(w_{\alpha_{1}}, w_{\alpha_{2}} \cdots w_{\alpha_{l}} \lambda\right) \cdots M\left(w_{\alpha_{l}}, \lambda\right)$.

Then $w \mapsto M(w, \lambda)$ is a homomorphism, as is $w \mapsto M(w, \lambda)$ evaluated at some $U_{-\alpha}, \alpha$ simple. The above map $M(w, \lambda)$ is evaluated at $U_{-\alpha}, \alpha=e_{1}-e_{2}$.

We note that the linear independence of distinct characters of $\mathfrak{f}^{*}$ implies that the $M(w, \lambda)$ evaluated at $U_{-\alpha}$ are linearly independent for $w \in R$, and therefore the operators $\{\mathfrak{a}(w, \lambda) \mid w \in R\}$ are linearly independent.

2. Type $\mathrm{B}_{n} . \quad \Phi=\left\{ \pm e_{i} \pm e_{j}, \pm e_{k} \mid 1 \leqq i<j \leqq n, 1 \leqq k \leqq n\right\}$. The dual root system $\Phi^{v}=\left\{ \pm e_{i} \pm e_{j}, \pm 2 e_{k} \mid 1 \leqq i<j \leqq n, 1 \leqq k \leqq n\right\}$ is type $\mathrm{C}_{n}$. The Weyl group $W \cong S_{n} \ltimes Z_{2}^{n}$ acts on $\Phi$ and $\Phi^{v}$ by permutations and sign changes on the $e_{i}$.

THeOREM $\mathrm{B}_{n} . \quad R \cong \boldsymbol{Z}_{2} \times \cdots \times \boldsymbol{Z}_{2}$ with $|R|$ dividing both 2 and $\left[\mathfrak{f}^{*}:\left(\mathfrak{l}^{*}\right)^{2}\right]$.

Suppose $w=s c \in W_{\lambda}$ with $s \in S_{n}$ and $c \in Z_{2}^{n}$. We may replace $w$ by a conjugate under $S_{n}$ to assume the cycles in $s$ consist of consecutive integers, and then by a conjugate by a sign change to assume that $c$ changes the sign of at most one $e_{i}$ in each orbit of $s$.

LEMMA 1. If $w=s c \in R$, then a nontrivial cycle of $s$ can not have only one sign change associated to it.

Proof. We may assume the cycle is $(k k+1 \cdots n-1 n), k<n$, 
and that the sign change is on $2 e_{n}$. Repeated application of $w^{-1}$ sends $e_{n-1}-e_{n}$ to $e_{k}-e_{k+1}$, which $w^{-1}$ then sends to $-e_{n}-e_{k}$. Thus $e_{k}+e_{n-1} \in \mathscr{L}$.

If $k=n-1$, then $2 e_{n-1} \in \mathscr{L} \cap \Phi^{v}$. But then $2 e_{n-1}>0$ and $w^{-1}\left(2 e_{n-1}\right)=-2 e_{n}<0$ contradicts $w \in R$.

Otherwise $k<n-1$ and $e_{k}+e_{n-1} \in \mathscr{L} \cap \Phi^{v}$. But then $e_{k}+e_{n-1}>0$ and $w^{2}\left(e_{k}+e_{n-1}\right)=w\left(e_{k+1}+e_{n}\right)=e_{k+2}-e_{k}<0$ contradicts $w \in R$.

LeMma 2. Any nontrivial cycle of $s \in S_{n}$ must be a transposition if $w=s c \in R$.

Proof. We may assume that the cycle is $(k \cdots n-1 n)$, and by the above lemma, that there are no sign changes associated to this cycle, i.e., $c\left(2 e_{i}\right)=2 e_{i}$ for $k \leqq i \leqq n$.

Then $w\left(e_{n-1}+e_{n}\right)=e_{n}+e_{k}$ implies that $e_{k}-e_{n-1} \in \mathscr{L}$. If $k<n-1$, then $e_{k}-e_{n-1} \in \mathscr{L} \cap \Phi^{v}$, with $e_{k}-e_{n-1}>0$ and $w^{-1}\left(e_{k}-e_{n-1}\right)=e_{n}-e_{n-2}<0$, contradicting $w \in R$. Thus $k=n-1$ and the cycle is a transposition.

By the two lemmas, any $w=s c \in R$ is conjugate to a product of disjoint transpositions and sign changes, so $w^{2}=1$ and $R \cong$ $\boldsymbol{Z}_{2} \times \cdots \times \boldsymbol{Z}_{2}$. Further, no such $w \neq 1$ can fix an $e_{i}$. This follows by the argument for type $\mathrm{A}_{n}$ if $s \neq 1$. If $s=1, w=c \neq 1$ changes the sign of some $e_{j}$. Then if $w\left(e_{i}\right)=e_{i}$ we have $e_{j}-e_{i}-w\left(e_{j}-e_{i}\right)=$ $2 e_{j} \in \mathscr{L} \cap \Phi^{v}$. But then $2 e_{j}>0$ and $w\left(2 e_{j}\right)<0$ contradicts $w \in R$.

Thus $R$ permutes $\left\{ \pm e_{i} \mid 1 \leqq i \leqq n\right\}$ with $\operatorname{stab}_{R}\left( \pm e_{i}\right)=\{1\}$, so $|R|$ divides $2 n$.

We now have that any $w \in R$ is conjugate to one of 1 , (12) (34) $\cdots(n-1 n),(12) \cdots(k-1 k) c_{k+1} \cdots c_{n}$, or $c_{1} c_{2} \cdots c_{n}$, where $c_{i}$ is the sign change on $e_{i}$.

If we evaluate $M(w, \lambda)$ at $U_{-\alpha}, \alpha=e_{1}-e_{2}$, we get the homomorphism $c_{1} \cdots c_{n} \mapsto 2 e_{1}$ (i.e., $\lambda e_{1}$ ) and $w \mapsto e_{i}-w e_{i}$ if $w=s c$ with $s(i) \neq i$. We note that none of these characters can be trivial if $w \in R$, so $w \mapsto e_{i}-w e_{i}$ is an injective homomorphism from $R$ into the group of characters of $\mathfrak{t}^{*}$ generated by those of order 2 . Thus

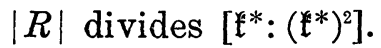

Of course, one may directly check that $w \mapsto e_{i}-w e_{i}$ is independent of $i$ and is an injective homomorphism from $R$ into the subgroup $Z\left[\Phi^{v}\right] / \mathscr{L}$ of $\left(\mathfrak{l}^{*}\right)^{\wedge}$ without reference to $M(w, \lambda)$.

We note that if $\mathfrak{t}=\boldsymbol{R}$, then $|R|=1$ or 2 , and that if $\mathfrak{t}$ is nonArchimedean with odd residual characteristic, then $|R|=1,2$, or 4 .

3. Type $\mathrm{C}_{n}$. $\Phi=\left\{ \pm e_{i} \pm e_{j}, \pm 2 e_{k} \mid 1 \leqq i<j \leqq n, 1 \leqq k \leqq n\right\}$. The dual root system $\Phi^{v}=\left\{ \pm e_{i} \pm e_{j}, \pm e_{k} \mid 1 \leqq i<j \leqq n, 1 \leqq k \leqq n\right\}$ is type $\mathrm{B}_{n}$. The Weyl group $W \cong S_{n} \ltimes \boldsymbol{Z}_{2}^{n}$ acts on $\Phi$ and $\Phi^{v}$ by permutations and sign changes on the $e_{i}$. 
THEOREM $\mathrm{C}_{n} . \quad R \cong \boldsymbol{Z}_{2} \times \boldsymbol{Z}_{2} \times \cdots \times \boldsymbol{Z}_{2}$ with the number of factors of $\boldsymbol{Z}_{2}$ bounded by $n$ and by $\left[\mathfrak{k}^{*}:\left(\mathfrak{k}^{*}\right)^{2}\right]-1$.

Suppose $w=s c \in W_{\lambda}, s \in S_{n}$ and $c \in \boldsymbol{Z}_{2}^{n}$. We may replace $w$ by a conjugate under a sign change to assume that $c$ changes the sign of at most one $e_{i}$ in each orbit of $s$.

Lemma 1. Suppose $w=s c \in R, s \in S_{n}, c \in \boldsymbol{Z}_{2}^{n}$. Then $s=1$.

Proof. If $s$ has a nontrivial cycle, by conjugation we may assume it is $(k \cdots n-1 n)$ and that $c$ changes the sign of at most one $e_{i}$ in the corresponding orbit.

Suppose $c\left(e_{i}\right)=e_{i}$ for $k \leqq i<n$ and $c\left(e_{n}\right)=-e_{n}$. Then $w^{-1}\left(e_{n}\right)=$ $e_{n-1}$ implies that $e_{n-1}-e_{n} \in \mathscr{L} \cap \Phi^{v}$. But repeated application of $w^{-1}$ sends $e_{n-1}-e_{n}$ to $e_{k}-e_{k+1}$, which $w^{-1}$ sends to $-e_{n}-e_{k}<0$, contradicting $w \in R$.

Now suppose $c\left(e_{i}\right)=e_{i}$ for $k \leqq i \leqq n$. Then $w^{-1}\left(e_{n}\right)=e_{n-1}$ and $e_{n-1}-e_{n} \in \mathscr{L} \cap \Phi^{v}$. But then $w\left(e_{n-1}-e_{n}\right)=e_{n}-e_{k}<0$ contradicts $w \in R$.

Thus $s=1$ if $w=s c \in R$, and $R$ is contained in the group of sign changes in $W$. Hence $R \cong Z_{2} \times \cdots \times Z_{2}$ with the number of factors bounded by $n$.

Let $w \in R$. By conjugation we may assume that $w=c_{l k} c_{k+1} \cdots c_{n}$.

Lemma 2. If $c_{k} c_{k+1} \cdots c_{n} \in R$, then $c_{i} \in R, k \leqq i \leqq n$.

Proof. $e_{i}$ and $e_{i}-e_{j}, k \leqq i \neq j \leqq n$, correspond to characters of order 2 , and $\mathscr{L}$ contains $Z\left[2 e_{i} \mid k \leqq i \leqq n\right]$. Then $\alpha-c_{i} \alpha \in \mathscr{L}$ for all simple $\alpha$, so $c_{i} \in W_{\lambda}, k \leqq i \leqq n$. Since $R\left(c_{i}\right) \leqq R\left(c_{k} \cdots c_{n}\right)$ does not intersect $\Delta^{\prime}$, we have that $c_{i} \in R$. (Recall that $R(w)=$ $\{\alpha>0 \mid w \alpha<0\}$.)

Thus any $R$ is conjugate to $\left\langle c_{k}, c_{k+1}, \cdots, c_{n}\right\rangle$ for some $k, 1 \leqq k \leqq n$, taking $c_{k} \cdots c_{n}$ above with as many sign changes as possible.

Note that each $e_{i}$ corresponds to a character of order $2, k \leqq i \leqq n$, and that these characters must be distinct, since $e_{i}-e_{j}$ does not correspond to the trivial character, $k \leqq i \neq j \leqq n$. Conversely, we may define a character $\lambda$ with $R$-group $\left\langle c_{k}, c_{k+1}, \cdots, c_{n}\right\rangle$ by assigning a distinct character of order 2 to each $e_{i}, k \leqq i \leqq n$.

Thus the number of factors of $Z_{2}$ in $R$ is bounded by $\left[\mathfrak{f}^{*}:\left(\mathfrak{l}^{*}\right)^{2}\right]-1$.

Note that there can be more reducibility in the case of type $\mathrm{C}_{n}$ than in the case of type $\mathrm{B}_{n}$.

$\mathrm{B}_{n}:|R|$ divides $2 n$ and $\left[\mathfrak{k}^{*}:\left(\mathfrak{k}^{*}\right)^{2}\right]$.

$\mathrm{C}_{n}:|R|$ divides $2^{n}$ and $2^{\left[*^{*} ;\left(*^{*}\right)^{2}\right]-1}$. 
If $\mathfrak{t}=\boldsymbol{R}$, we again get $|R|=1$ or 2 .

4. Type $\mathrm{D}_{n} . \quad \Phi=\Phi^{v}=\left\{ \pm e_{i} \pm e_{j} \mid 1 \leqq i<j \leqq n\right\}$ is self-dual and the Weyl group $W \cong S_{n} \ltimes \boldsymbol{Z}_{2}^{n-1}$ acts as permutations and even sign changes on the $e_{i}$.

THEOREM $\mathrm{D}_{n}$.

(a) Suppose $n$ is even. Then if $R$ is abelian, $R \cong Z_{2} \times$ $Z_{2} \times \cdots \times Z_{2}$ with the number of factors bounded by $n-1$ and by $\left[\mathfrak{\mathfrak { t }}^{*}:\left(\mathfrak{\mathfrak { t }}^{*}\right)^{2}\right]-1$. If $R$ is nonabelian, then $R \cong\left(\boldsymbol{Z}_{2} \times \cdots \times \boldsymbol{Z}_{2}\right) \times$ $\left(\boldsymbol{Z}_{2} \times \boldsymbol{Z}_{2} \times \cdots \times \boldsymbol{Z}_{2}\right)$ with the order of the first factor dividing both $2 n$ and $\left[\mathfrak{k}^{*}:\left(\mathfrak{E}^{*}\right)^{2}\right]$, and the number of factors of $\boldsymbol{Z}_{2}$ in the normal subgroup an odd number bounded by $n-1$ and $\left[\mathfrak{f}^{*}:\left(\mathfrak{t}^{*}\right)^{2}\right]-1$.

(b) Suppose $n$ is odd. Then if $R$ is abelian, $R \cong \boldsymbol{Z}_{2} \times \cdots \times \boldsymbol{Z}_{2}$ with the number of factors of $\boldsymbol{Z}_{2}$ bounded by $n-1$ and $\left[\mathfrak{E}^{*}:\left(\mathfrak{E}^{*}\right)^{2}\right]-1$, or $R=\boldsymbol{Z}_{4}$. If $R$ is nonabelian, $R \cong \boldsymbol{Z}_{4} \ltimes\left(\boldsymbol{Z}_{2} \times \cdots \times \boldsymbol{Z}_{2}\right)$ with the number of factors of $\boldsymbol{Z}_{2}$ in the normal subgroup an even number bounded by $n-3$ and $\left[\mathfrak{t}^{*}:\left(\mathfrak{k}^{*}\right)^{2}\right]-2$.

The actions on the normal factors of the semi-direct products are described explicitly in the course of the proof.

Lemma 1. Suppose $w=s c \in R, s \in S_{n}$ and $c \in Z_{2}^{n-1}$. Then $s^{2}=1$.

Proof. Suppose $s$ has a cycle of length $\geqq 3$. Replacing $w$ by a conjugate under $S_{n}$, we may assume the cycle is $(k k+1 \cdots n)$, $k<n-1$. Then by conjugating $w$ by a sign change, we may assume that $c$ changes the sign of at most 2 of the $e_{i}$ in each orbit of $s$.

If $c$ involves no sign changes on $e_{k}, \cdots, e_{n}$, then $w^{-1}\left(e_{n-1}+e_{n}\right)=$ $e_{n-2}+e_{n-1}$ implies that $e_{n-2}-e_{n} \in \Delta^{\prime}$. But then $w\left(e_{n-2}-e_{n}\right)<0$ contradicts $w \in R$.

If $c$ involves only one sign change on $e_{k}, \cdots, e_{n}$, we may suppose it is on $e_{n}$. Then $w\left(e_{n-1}+e_{n}\right)=e_{n}-e_{k}$ implies $e_{k}+e_{n-1} \in \Delta^{\prime}$. But then $w^{2}\left(e_{k}+e_{n-1}\right)=e_{k+2}-e_{k}<0$ contradicts $w \in R$.

Finally, if there are two sign changes involved, we may suppose they are on $e_{n-1}$ and $e_{n}$. Then $w\left(e_{n-1}-e_{n}\right)=-e_{n}+e_{k}$ implies that $e_{k}-e_{n-1} \in \Delta^{\prime}$. But then $w^{-1}\left(e_{k}-e_{n-1}\right)=-e_{n}-e_{n-2<0}$ contradicts $w \in R$.

Note that $w=s c \in R, s^{2}=1$ implies that $w^{2}=\left(s c s^{-1}\right) c$ is a sign change in $R$ and thus $w^{4}=1$. If we let $R^{\prime}$ be the group of sign changes in $R$, then $R^{\prime} \leqq R$ and $R / R^{\prime} \cong Z_{2} \times \cdots \times \boldsymbol{Z}_{2}$.

Lemma 2. Suppose $c_{k} c_{k+1} \cdots c_{n} \in R$ with $k>1$. Then $R$ contains all even sign changes on $\left\{e_{k}, e_{k+1}, \cdots, e_{n}\right\}$. 
Proof. If $k>1$, then $c_{k} c_{k+1} \cdots c_{n} \in R$ if and only if $e_{i}-e_{j}$ corresponds to a character of order 2 for $k \leqq i<j \leqq n$, and $e_{n-1} \pm e_{n}$ correspond to the same character. Then $c_{i} c_{i+1} \in W_{\lambda}$ and $R\left(c_{i} c_{i+1}\right) \subseteq$ $R\left(c_{k} c_{k+1} \cdots c_{n}\right)$ imply that $c_{i} c_{i+1} \in R$, for $k \leqq i<n$.

Thus $R^{\prime}$ consists of all even sign changes on $\left\{e_{k}, \cdots, e_{n}\right\}$, and $\left|R^{\prime}\right| \leqq 2^{n-1}$.

Further, since the characters corresponding to $e_{i}-e_{j}$ are nontrivial for $k \leqq i<j<n$, the characters corresponding to $e_{i}-e_{n}$ are distinct, $k \leqq i<n$. Thus $\left|R^{\prime}\right| \leqq 2^{\left[t^{* *}\left(i^{*}\right)^{2}\right]-1}$.

Now, suppose $w=s c \in R$ with $s \neq 1$. By conjugation we may assume $s=(12)(34) \cdots(k-1 k)$ with $k \leqq n$. Then $c\left(e_{i}\right)=-e_{i}$ for $k<i \leqq n$; first, we may assume $c\left(e_{k}\right)=+e_{k}$ by conjugation by $c_{k} c_{n}$ if necessary. Then if $c\left(e_{i}\right)=e_{i}$ for some $k<i \leqq n, w\left(e_{k}-e_{i}\right)=$ $e_{k-1}-e_{i}$ would imply $e_{k-1}-e_{k} \in \Delta^{\prime}$. But then $w\left(e_{k-1}-e_{k}\right)<0$ contra$\operatorname{dicts} w \in R$. Thus we have shown

Lemma 3. $\operatorname{stab}_{R}\left( \pm e_{i}\right) \leqq R^{\prime}$.

Further, any element of order 2 in $R$ is conjugate to one of $c \in \boldsymbol{Z}_{2}^{n-1}$, (12) (34) $\cdots(n-1 n)$, (12) (34) $\cdots(n-1 n) c_{n-1} c_{n}$, or (12) $\cdots$ $(k-1 k) c_{k+1} \cdots c_{n}$ for some $k$. Any element of order 4 in $R$ is conjugate to (12) (34) $\cdots(m-1 m) c_{k} c_{k+2} \cdots c_{m} c_{m+1} c_{m+2} \cdots c_{n}$ for some $m, k$ with $2 \leqq k \leqq m \leqq n$, where the sign change changes the sign of the $e_{i}, m<i \leqq n$ and of the $e_{j}, j$ even, $k \leqq j \leqq m$.

If $R$ has no elements of order 4 , then $R \cong \boldsymbol{Z}_{2} \times \cdots \times \boldsymbol{Z}_{2}$ is abelian. Suppose there is an element of order 4. We distinguish the cases $n$ even and $n$ odd.

Case 1. Suppose $n$ is even.

Then any element of $R$ of order 4 is conjugate to $w=$ (12) (34) $\cdots(m-1 m) c_{k} c_{k+2} \cdots c_{m} \cdot c_{m+1} \cdots c_{n}$ with $k \leqq m-2$. Suppose for a moment that $m=n$. Then since $w \in W_{\lambda}$ if and only if $\alpha-$ $w \alpha \in \mathscr{L}$, the $\lambda_{\alpha}$ must satisfy relations corresponding to $e_{1}-e_{2} \equiv$ $e_{3}-e_{4} \equiv \cdots \equiv e_{n-1}-e_{n} \equiv e_{n-1}+e_{n}, 2\left(e_{1}-e_{2}\right) \equiv 0$, and $2\left(e_{i}-e_{j}\right) \equiv 0$ $\bmod \mathscr{L}$ for $k \leqq i<j \leqq n$. Further, if $w \in R$ then $\mathscr{L} \cap\left\{e_{i}-e_{j} \mid k-\right.$ $1 \leqq i<j \leqq n\}=0$, and thus $\lambda$ is given by

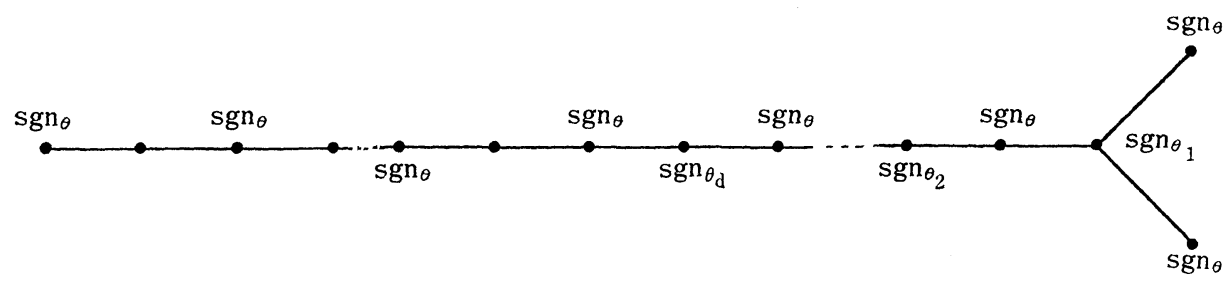

where $k=n-2 d$. 
Since $w^{2}=c_{k-1} c_{k} \cdots c_{n} \in R$, by Lemma $2, R$ contains the group $\boldsymbol{Z}_{2}^{2 d+1}$ of all even sign changes on $\left\{e_{k-1}, e_{k}, \cdots, e_{n}\right\}$. Then (12) (34) $\cdots$ $(n-1 n) \in R$ and $R \geqq\langle(12)(34) \cdots(n-1 n)\rangle \ltimes\left\langle c_{n} c_{n-1}, c_{n-1} c_{n-2}, \cdots\right.$, $\left.c_{k} c_{k-1}\right\rangle$ is nonabelian.

If there are other sign changes in $R$, we may assume they involve $e_{l}, e_{l+1}, \cdots, e_{k-2}$, where $l$ is odd. Then the group $R^{\prime}$ of all sign changes in $R$ consists all even sign changes on $\left\{e_{l}, e_{l+1}, \cdots, e_{n}\right\}$ and each $e_{i}-e_{j}, l \leqq i<j \leqq n$ corresponds to a character of order 2 .

Now, if $m<n, w=(12) \cdots(m-1 m) c_{k} c_{k+2} \cdots c_{m} . \quad c_{m+1} \cdots c_{n}$ acts on $D_{m}=\left\{ \pm e_{i} \pm e_{j} \mid 1 \leqq i<j \leqq m\right\} \subset \Phi$ as the above. Then $e_{1}-e_{2} \equiv e_{3}-e_{4} \equiv \cdots \equiv e_{m-1}-e_{m} \equiv e_{m-1}+e_{m} \bmod \mathscr{L}$, and $e_{i}-e_{j}$ corresponds to a character of order 2 for $k-1 \leqq i<j \leqq m$ and for $m+1 \leqq i<j \leqq n$.

Thus $e_{m-1}-e_{m} \equiv\left(e_{n-1}-e_{n}\right)+\left(e_{n-1}+e_{n}\right) \not \equiv 0$ and $2\left(e_{m}-e_{m+1}\right) \equiv$ $e_{m-1}-e_{m} \bmod \mathscr{L}$, and $\lambda$ is given by

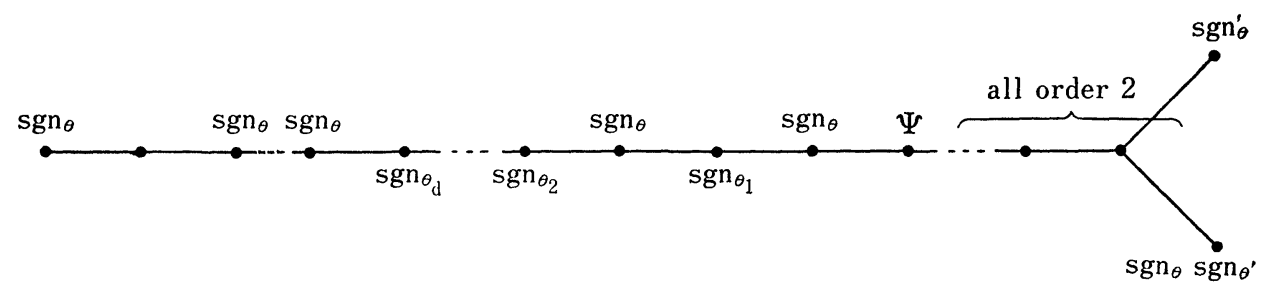

with $\Psi^{2}=\operatorname{sgn}_{\theta}$.

Again, $R$ contains $s c=(12) \cdots(m-1 m) c_{m+1} \cdots c_{n}$ and we may assume by conjugation the $R^{\prime}$ consists of all even sign changes on $\left\{e_{l}, \cdots, e_{m}\right\}$.

Then $R \geqq\langle s c\rangle \ltimes R^{\prime}$ is nonabelian, as before. Suppose there are other $s^{\prime} c^{\prime}$ in $R$. If $s^{\prime}=s$ then $s c^{\prime}$ is in the subgroup $\langle s c\rangle \ltimes R^{\prime}$. If $s^{\prime} \neq s$ then $R \geqq\left\langle s c, s^{\prime} c^{\prime}\right\rangle \ltimes R^{\prime}$. We keep adding new elements of $R$ until

Lemma 4. $R=\left\{1, s c, s^{\prime} c^{\prime}, \cdots\right\} \ltimes R^{\prime}$ with the permutations $s^{(i)}$ distinct.

Further, the order of the first subgroup divides $2 n$ by Lemma 3 , and also $R^{\prime} \leqq Z_{2}^{n-1}$. Thus $|R|$ divides $n \cdot 2^{n}$.

Formally define a character corresponding to $2 e_{n}$ to be $-\left(e_{n-1}-\right.$ $\left.e_{n}\right)+\left(e_{n-1}+e_{n}\right)$ and then use $2 e_{i}=2\left(e_{i}-e_{n}\right)+2 e_{n}$ to define a character corresponding to $2 e_{i}$. If $w=s c \in R, c \in Z_{2}^{n-1}$ with $s \neq 1$, then $e_{i}-w e_{i}$ is a character of order 2 and $w \mapsto e_{i}-w e_{i}$ is an injective homomorphism on the first (nonnormal) factor of $R$. Thus the order of this factor divides $\left[\mathfrak{t}^{*}:\left(\mathfrak{t}^{*}\right)^{2}\right]$.

We have already seen that the number of factors of $\boldsymbol{Z}_{2}$ in $R^{\prime}$ is bounded by $n-1$ and $\left[\mathfrak{t}^{*}:\left(\mathfrak{k}^{*}\right)^{2}\right]-1$. 
Finally, if $n$ is even and $R$ is abelian, we may write $R=\left\{1, s c, s^{\prime} c^{\prime}, \cdots\right\} \times R^{\prime} \cong Z_{2} \times \cdots \times Z_{2}$ as in Lemma 4 , with the $s^{(i)}$ distinct. We show that the number of factors of $\boldsymbol{Z}_{2}$ is bounded by $n-1$ and $\left[\mathfrak{F}^{*}:\left(\mathfrak{E}^{*}\right)^{2}\right]-1$. If $R=R^{\prime}$, this is true. If $R^{\prime}=\{1\}$, then

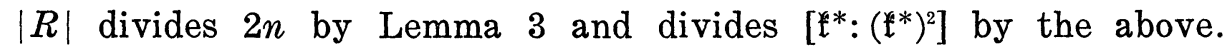
Suppose that both factors are nontrivial and that $R$ is abelian. We may assume that (12) (34) $\cdots(n-1 n)$ or $(12) \cdots(k-1 k) c_{k+1} \cdots c_{n}$ is in $R$.

Suppose that (12) $\cdots(k-1 k) c_{k+1} \cdots c_{n}$ and $c_{l} \cdots c_{k-1} c_{k} \in R, k<n$. Then also $c_{k-1} c_{k} \in R$. Then if $s^{\prime} c^{\prime} \in R, s^{\prime} \neq 1$, (12) $\cdots(k-1 k)$, we may assume that $c^{\prime}\left(e_{i}\right)=e_{i}, i=k-1, k$. Then $s^{\prime}(k) \neq k-1$ by Lemma 3 and $s^{\prime} c^{\prime}$ does not commute with $c_{k-1} c_{k}$, contradicting the assumption that $R$ is abelian. Thus no other $s^{\prime} c^{\prime}$ are in $R$. Further, if $l<k-1$, then $c_{k-2} c_{k-1} \in R$, contradicting $R$ abelian, and $c_{k-1} c_{k}$ is the only sign change in $R$. Thus $|R|=4$.

Suppose that (12) (34) $\cdots(n-1 n)$ and $c_{i} \cdots c_{n-1} c_{n} \in R$. Then if $1<i<n-1, c_{n-2} c_{n-1} \in R$, contradicting the assumption $R$ is abelian. Thus $i=1$ or $n-1$. If $i=1$, the $R^{\prime}=\left\{1, c_{1} c_{2} \cdots c_{n}\right\}$ and $|R|$ divides $2 n$ and $2\left[\mathfrak{f}^{*}:\left(\mathfrak{k}^{*}\right)^{2}\right]$. If $i=n-1$, then $|R|=4$, as above.

We note that if $\mathfrak{k}=\boldsymbol{R}$, then one can have $|R|=1,2$, or 4 in the case of $\mathrm{D}_{n}, n$ even.

Case 2. Suppose $n$ is odd.

In this case any element of order 4 in $R$ is conjugate to $w=$ (12) $\cdots(m-1 m) . \quad c_{k} c_{k+2} \cdots c_{m} \cdot c_{m+1} \cdots c_{n}$, with $k \leqq m<n$.

If $m=n-1$ and $w=(12) \cdots(n-2 n-1) c_{n-1} c_{n}$, then $\lambda$ is given by

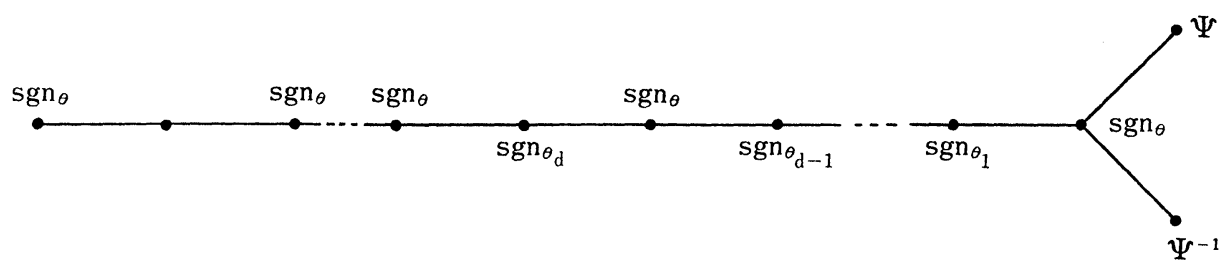

with $\Psi^{2}=\operatorname{sgn}_{0}$.

Then $w^{2}=c_{k-1} c_{k} \cdots c_{n-1} \in R$ and thus $R$ contains all even sign changes on $\left\{e_{k-1}, e_{k}, \cdots, e_{n-1}\right\}$. Thus each $e_{i}-e_{j}, k-1 \leqq i<j \leqq$ $n-1$ corresponds to a character of order 2 and also $s c=(12) \cdots$ $(n-2 n-1) c_{n-1} c_{n} \in R$. We have $R \geqq\langle s c\rangle \ltimes\left\langle c_{k-1} c_{k}, c_{k} c_{k+1}, \cdots\right.$, $\left.c_{n-3} c_{n-2}\right\rangle \cong \boldsymbol{Z}_{4} \ltimes \boldsymbol{Z}_{2}^{n-k-1}$.

If $m<n-1$ and $w=(12) \cdots(m-1 m) c_{k} c_{k+2} \cdots c_{m} c_{m+1} \cdots c_{n} \in R$, then $\lambda$ is given by 


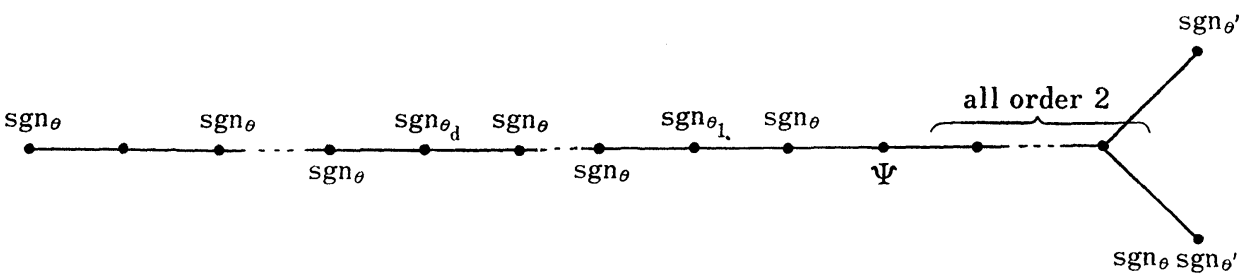

with $\Psi^{2}=\operatorname{sgn}_{\theta}$.

In this case, $w^{2}=c_{k-1} c_{k} \cdots c_{m} \in R$ and $R$ contains all even sign changes on $\left\{e_{k-1}, \cdots, e_{m}\right\}$. Then $s c=(12) \cdots(m-1 m) c_{m} c_{m+1} \cdots c_{n} \in R$ and each $e_{i}-e_{j}, k-1 \leqq i<j \leqq m$, and $m+1 \leqq i<j \leqq n$, corresponds to a character of order 2 . Also $2\left(e_{m}-e_{m+1}\right) \equiv e_{m-1}-e_{m} \equiv$ $\left(e_{n-1}-e_{n}\right)+\left(e_{n-1}+e_{n}\right) \bmod \mathscr{L}$.

$R \geqq\langle s c\rangle \ltimes\left\langle c_{k-1} c_{k}, c_{k} c_{k+1}, \cdots, c_{m-2} c_{m-1}\right\rangle=Z_{4} \ltimes Z_{2}^{m-k}$. Let $R^{\prime \prime}$ be the group of the even sign changes on $\left\{e_{1}, e_{2}, \cdots, e_{m-1}\right\}$ which occur in $R$. Then $R=\langle s c\rangle \ltimes R^{\prime \prime}$ by

Lemma 5. If $s^{\prime} c^{\prime} \in R$, then $s^{\prime}=1$ or $s^{\prime}=s=(12) \cdots(m-1 m)$.

Proof. If $s c$ and $s^{\prime} c^{\prime}$ are in $R$, then $s^{\prime} s=s s^{\prime}$ by Lemma 1 , so that $s^{\prime}$ permutes the odd number of fixed points $m+1, \cdots, n$ of $s$. $s^{\prime}$ must permute them faithfully by Lemma 3 . But this contradicts Lemma 1 , which implies that $s^{\prime}$ must be a product of transpositions.

Thus $R=\langle s c\rangle \ltimes R^{\prime \prime} \cong Z_{4} \ltimes R^{\prime \prime}$.

Since $R^{\prime \prime} \leqq \boldsymbol{Z}_{2}^{m-2} \leqq \boldsymbol{Z}_{2}^{n-3}$, the number of factors of $\boldsymbol{Z}_{2}$ in $R^{\prime \prime}$ is bounded by $n-3$. It is also bounded by $\left[\mathfrak{t}^{*}:\left(\mathfrak{t}^{*}\right)^{2}\right]-2$, since the number of factors of $Z_{2}$ in $R^{\prime}=\left\langle c_{m-1} c_{m}\right\rangle \times R^{\prime \prime}$ is bounded by $\left[\mathfrak{f}^{*}:\left(\mathfrak{l}^{*}\right)^{2}\right]-1$.

Finally, suppose that $R$ is abelian and $n$ is odd. Then either there are no factors of $\boldsymbol{Z}_{2}$ above, i.e., $k=m, R^{\prime \prime}=\{1\}$ and $R \cong \boldsymbol{Z}_{4}$, or $R$ is contained in the group $\boldsymbol{Z}_{2}^{n-1}$ of even sign changes.

\section{Type $\mathrm{E}_{8}$.}

THEOREM $\mathrm{E}_{6} . \quad R \cong 1, \boldsymbol{Z}_{2}, \boldsymbol{Z}_{3}, \boldsymbol{Z}_{3} \times \boldsymbol{Z}_{3}$ or $\boldsymbol{Z}_{6}$. Further, $\boldsymbol{Z}_{3} \times \boldsymbol{Z}_{3}$ occurs as a reducibility group if and only if $p=3$ or 3 divides $q-1$.

Arrange the simple roots in the traditional Dynkin diagram

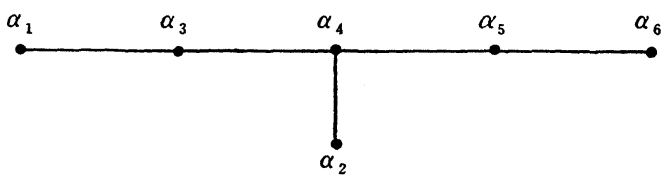


The roots spanned by $\left\{\alpha_{2}, \alpha_{3}, \alpha_{4}, \alpha_{5}, \alpha_{6}\right\}$ form a subsystem of type $\mathrm{D}_{5}$, giving an inclusion of Weyl groups $W\left(D_{5}\right)<W\left(E_{6}\right)$. Comparing orders, $2^{7} \cdot 3 \cdot 5$ and $2^{7} \cdot 3^{4} \cdot 5$ respectively, we see that a 2 -Sylow subgroup of $W\left(D_{5}\right)$ is also a 2-Sylow subgroup of $W\left(E_{6}\right)$. By conjugation, we may assume that a 2-Sylow subgroup of $W_{\lambda}$ is contained in $W\left(D_{5}\right)$.

Let $\alpha_{8}=e_{1}-e_{2}, \cdots, \alpha_{3}=e_{4}-e_{5}$ and $\alpha_{2}=e_{4}+e_{5}$. Then by our $\mathrm{D}_{5}$ results, potential candidates in $R \cap W\left(D_{5}\right)$ are conjugate to $c_{2} c_{3} c_{4} c_{5}$, $c_{4} c_{5}$, (12) $c_{2} c_{3} c_{4} c_{5}$, or (12) (34) $c_{4} c_{5}$. Adding the condition $w \alpha_{1} \equiv \alpha_{1}$, only $c_{2} c_{3} c_{4} c_{5}=w_{\alpha_{5}} w_{\alpha_{2}+\alpha_{3}+2 \alpha_{4}+\alpha_{5}} w_{\alpha_{3}} w_{\alpha_{2}}$ can be in an $E_{8} R$-group.

If $c_{2} c_{3} c_{4} c_{5} \in R$, then $\lambda$ is given by

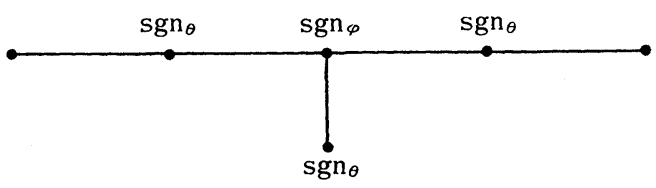

with $\operatorname{sgn}_{\theta} \neq \operatorname{sgn}_{\varphi}$. There can be no other elements of order 2 in $R$ with $c_{2} c_{3} c_{4} c_{5}$; if there were another, by conjugation we could assume it is $c_{1} c_{2} c_{3} c_{4}$. But the product $c_{1} c_{5}$ cannot be in an $R$-group.

Thus, if there is an element with order a power of 2 in $R$, it has order 2 and is unique, hence is in the center of $R$.

Note that the longest Weyl element $w_{0}$ and the character

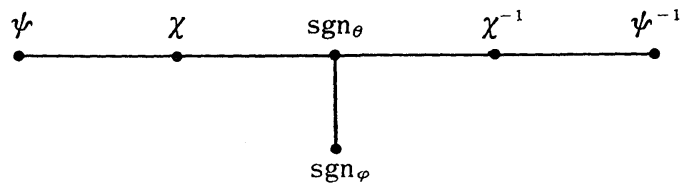

are conjugate to the above.

There is only one conjugacy class of elements of order 5 in $W\left(E_{6}\right)$ and none of its elements can be in an $R$-group. Thus, $R$ is the direct product of a 2-Sylow subgroup ( 1 or $Z_{2}$ ) and a 3-Sylow subgroup. Examining conjugacy classes of elements of order 3 or 9, [5], any element in $R$ with order a power of 3 is conjugate to one of $w_{\alpha_{1}} w_{\alpha_{3}} w_{\alpha_{6}} w_{\alpha_{5}}$ or $w_{\alpha_{1}} w_{\alpha_{3}} w_{\alpha_{5}} w_{\alpha_{6}} w_{\alpha_{2}} w_{2}^{12321}$, where ${ }_{2}^{12321}$ represents the root $\alpha_{1}+2 \alpha_{2}+2 \alpha_{3}+3 \alpha_{4}+2 \alpha_{5}+\alpha_{8}$.

For $w_{\alpha_{1}} w_{\alpha_{3}} w_{\alpha_{6}} w_{\alpha_{5}} \in R, \lambda$ is given by

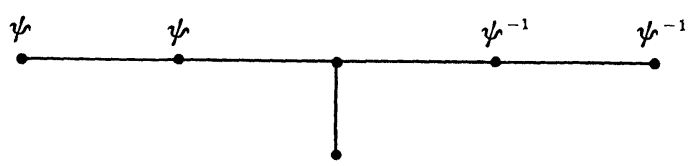

with $\psi$ of order 3 , giving $R \cong Z_{3}$. Further, if $\lambda_{\alpha_{2}}=\psi$ and $\lambda_{\alpha_{4}} \neq \psi^{ \pm 1}$ have order 3 , then $R=\left\langle w_{\alpha_{1}} w_{\alpha_{3}} w_{\alpha_{6}} w_{\alpha_{5}}, w_{\alpha_{1}} w_{\alpha_{3}} w_{\alpha_{2}} w_{12321}\right\rangle \cong Z_{3} \times Z_{3}$. If instead $\lambda_{\alpha_{2}} \neq \lambda_{\alpha_{4}}$ have order 2 , then $R=\left\langle w_{\alpha_{1}} w_{\alpha_{3}} w_{\alpha_{6}} w_{\alpha_{5}}, w_{0}\right\rangle \cong \boldsymbol{Z}_{6}$. 
For $w_{\alpha_{1}} w_{\alpha_{3}} w_{\alpha_{\tilde{j}}} w_{\alpha_{6}} w_{\alpha_{2}} w_{2}^{12221} \in R, \lambda$ is given by

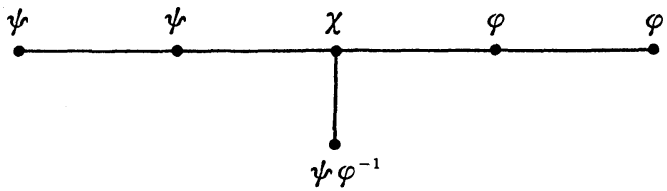

with each character having order $3, \psi \neq \phi$ and $\chi \notin\langle\psi, \varphi\rangle$. Then $R \cong \boldsymbol{Z}_{3}$, or there is also an element of type $2 \mathrm{~A}_{2}$ [5] in $R \cong \boldsymbol{Z}_{3} \times \boldsymbol{Z}_{3}$, and we are in one of the above cases.

Note that if $G$ is a Chevalley group over $k=R$, then $R=1$ and $\operatorname{Ind}_{B}^{G} \lambda$ is irreducible.

6. Type $\mathrm{E}_{7}$.

THEOREM $\mathrm{E}_{7} . \quad R$ may be nonabelian. If so, $R \cong$ dihedral group $D$ of order 8 , or $R \cong D \times Z_{2}$. $D \times Z_{2}$ can occur if and only if $p=2$ or 4 divides $q-1$.

If $R$ is abelian, then $R \cong \boldsymbol{Z}_{2}^{n}$ with $0 \leqq n \leqq 4, \boldsymbol{Z}_{3}, \boldsymbol{Z}_{4}$ or $\boldsymbol{Z}_{6}$. $\boldsymbol{Z}_{2}^{3}$ and $Z_{4}$ occur if and only if $p=2 . \quad Z_{2}^{4}$ occurs if and only if $\left[k^{*}:\left(k^{*}\right)^{2}\right] \geqq 16$. $\quad Z_{3}$ and $Z_{6}$ occur if and only if $p=3$ or 3 divides $q-1$.

Arrange the simple roots in the diagram

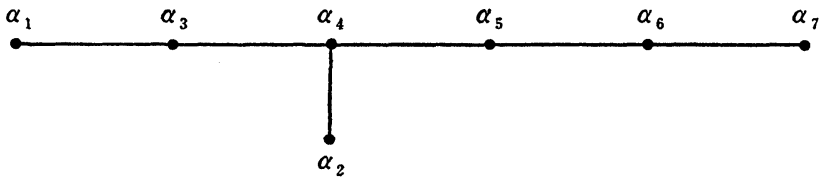

The roots $\left\{\alpha_{2}, \alpha_{3}, \alpha_{4}, \alpha_{5}, \alpha_{6}, \alpha_{7}\right\}$ span a subsystem of type $\mathrm{D}_{6}$, giving an inclusion of Weyl groups $W\left(D_{6}\right)<W\left(E_{7}\right)$. Let $w_{0}=-1$ be the longest Weyl element in $W\left(E_{7}\right)$. Comparing orders, $2^{9} \cdot 3^{2} \cdot 5$ and $2^{10} \cdot 3^{4} \cdot 5 \cdot 7,\left\langle w_{0}\right\rangle x$ a 2-Sylow subgroup of $W\left(D_{6}\right)$ will be a 2-Sylow subgroup of $W\left(E_{7}\right)$. We first classify 2-Sylow subgroups of $R$-groups.

Let $\alpha_{7}=e_{1}-e_{2}, \cdots, \alpha_{3}=e_{5}-e_{6}$, and $\alpha_{2}=e_{5}+e_{6}$. Using our $W\left(D_{6}\right)$ notation and grouping by $W\left(E_{7}\right)$-conjugacy classes, elements in $R$ with order a power of 2 are conjugate to:

$3 A_{1}:(12)(34)(56) c_{5} c_{6}, w_{0} c_{3} c_{4} c_{5} c_{6}$

$4 A_{1}: c_{3} c_{4} c_{5} c_{6}, w_{0}(12)(34)(56) c_{5} c_{6}$

$5 A_{1}:(12) c_{3} c_{4} c_{5} c_{6}, w_{0}(34)(56), w_{0} c_{5} c_{6}$

$6 A_{1}: c_{1} c_{2} c_{3} c_{4} c_{5} c_{6}, w_{0}(56)$

$7 A_{1}: w_{0}=-1$

$D_{4}\left(a_{1}\right)+2 A_{1}:(12)(34) c_{2} c_{4} c_{5} c_{6}, w_{0}(12)(34)(56) c_{4} c_{6}$

$2 A_{3}+A_{1}: w_{0}(12)(34) c_{2} c_{3}$. 
Suppose there is an element or order 4 in $R$. If it has type $\mathrm{D}_{4}\left(a_{1}\right)+2 A_{1}$, we may assume it is (12) $(34) c_{2} c_{4} c_{5} c_{6}$ and $\lambda$ is given by

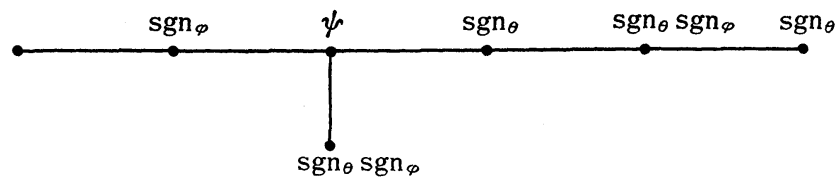

with $\psi^{2}=\operatorname{sgn}_{\theta} \neq \operatorname{sgn}_{\varphi} . \quad$ Then $R \geqq\left\langle(13)(24)(56) c_{2} c_{4}\right\rangle \ltimes\left\langle(12)(34) c_{2} c_{4} c_{5} c_{6}\right\rangle \cong$ dihedral group $D_{4}$.

If $\lambda_{\alpha_{1}}$ is "generic", then $R \cong D$ is nonabelian with order 8. If $R$ is larger, a consideration of other possible elements shows that we may assume, by conjugation, that $R$ contains one of $w_{0} c_{3} c_{4} c_{5} c_{6}$, $w_{0} c_{2} c_{4} c_{5} c_{6}$, or $w_{0} c_{1} c_{4} c_{5} c_{6}$. Each of these three cases occurs, giving $R \cong D \times Z_{2}$ nonabelian of order 16 .

In the first case, $\lambda_{\alpha_{1}}^{2}=\operatorname{sgn}_{\theta} \operatorname{sgn}_{\varphi}$, which can occur if, and only if $\left[k^{*}:\left(k^{*}\right)^{4}\right] \geqq 16$, i.e., $p=2$ or 4 divides $q-1$.

In the second case, $\lambda_{\alpha_{1}} \neq \operatorname{sgn}_{\theta}, \operatorname{sgn}_{\varphi}, \operatorname{sgn}_{\theta} \operatorname{sgn}_{\varphi}$ has order 2 , which can occur if, and only if $p=2$.

In the third case, $\lambda_{\alpha_{1}}^{2}=\operatorname{sgn}_{\theta}$, and $\lambda_{\alpha_{1}} \psi^{-1} \neq \operatorname{sgn}_{\theta}, \operatorname{sgn}_{\varphi}, \operatorname{sgn}_{\theta} \operatorname{sgn}_{\varphi}$ has order 2 , which occurs if, and only if $p=2$.

We may now suppose that $R$ contains no elements of type $D_{4}\left(a_{1}\right)+2 A_{1}$.

Suppose that $R$ contains $w_{0}(12)(34) c_{2} c_{3}$. Then $\lambda$ is given by

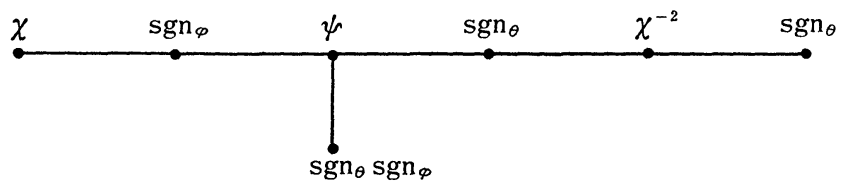

with $\Psi^{2}=\operatorname{sgn}_{\theta} \neq \operatorname{sgn}_{\varphi}, \chi$ of order 4 and $\chi^{2} \neq \operatorname{sgn}_{\theta}, \operatorname{sgn}_{\varphi}$. If $\chi^{2}=$ $\operatorname{sgn}_{\theta} \operatorname{sgn}_{\varphi}$, then we are in one of the above cases with (12) (34) $c_{2} c_{4} c_{5} c_{6} \in R$. Otherwise, $\chi^{2} \notin\left\langle\operatorname{sgn}_{\theta}, \operatorname{sgn}_{\varphi}\right\rangle$, so $p=2$, and then $R \cong \boldsymbol{Z}_{4}$.

If $R$ contains no elements of order 4, then a 2-Sylow subgroup of $R$ is a product of copies of $\boldsymbol{Z}_{2}$. An explicit list shows that $\boldsymbol{Z}_{2}$ occurs for any $k$, even the reals $\boldsymbol{R}$; that $\boldsymbol{Z}_{2}^{2}$ occurs for any nonArchimedean $k$ (we will need 2 characters of order 2); that $\boldsymbol{Z}_{2}^{3}$ occurs if, and only if $p=2$; and $Z_{2}^{4}$ occurs if, and only if $\left[k^{*}:\left(k^{*}\right)^{2}\right] \geqq 16$.

An easy calculation shows that $R$ can contain no elements of order 5 or 7 . Of elements of order a power of 3 , only conjugates of $w_{\alpha_{1}} w_{\alpha_{3}} w_{\alpha_{6}} w_{\alpha_{5}} w_{\alpha_{2}} w_{123210}\left(3 A_{2}\right)$ can be in an $R$-group. If this element is in $R$, then $\lambda$ is given by

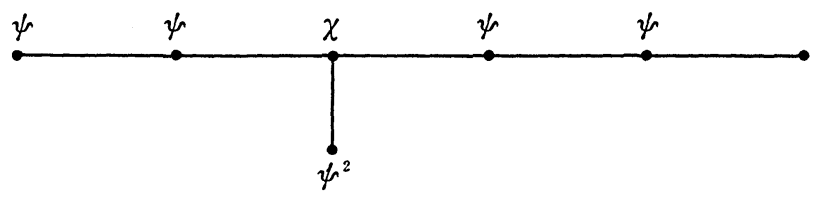


with $\psi \neq \chi^{ \pm 1}$ of order 3 . There are no other elements of order 3 in $R$ with this one, besides its inverse. Since we may specify only one character of order 2, there can be at most one element of order 2 in this $R$. Thus, $R \cong Z_{6}$. This does occur, with $R$ generated by an element of type $A_{5}+A_{2}$.

\section{Type $\mathrm{E}_{8}$.}

THeOREM $\mathrm{E}_{8}$. A nonabelian $R$-group will occur if and only if $\left[k^{*}:\left(k^{*}\right)^{2}\right] \geqq 16$. All nonabelian $R$ are conjugate to $\left\langle(12)(34)(56)(78) C_{7} C_{8}\right.$, (13) $\left.(24)(57)(68) C_{6} C_{8},(15)(26)(37)(48) C_{4} C_{8}\right\rangle \ltimes\left\langle C_{1} C_{3} C_{5} C_{7}, C_{2} C_{4} C_{6} C_{8}, C_{1} C_{2} C_{3} C_{4}\right.$, $\mathrm{C}_{3} \mathrm{C}_{4} \mathrm{C}_{5} \mathrm{C}_{6}>$.

If $R$ is abelian, then $R \cong \boldsymbol{Z}_{2}^{n}$ with $0 \leqq n \leqq 4, \boldsymbol{Z}_{4}, \boldsymbol{Z}_{4} \times \boldsymbol{Z}_{2}, \boldsymbol{Z}_{3}$, $\boldsymbol{Z}_{3} \times \boldsymbol{Z}_{3}$, or $\boldsymbol{Z}_{5}$.

$\boldsymbol{Z}_{2}^{n}$ occurs if and only if $\left[k^{*}:\left(k^{*}\right)^{2}\right] \geqq 2^{n+1}, 0 \leqq n \leqq 4 . \quad Z_{4}$ occurs if and only if $p=2$ or 4 divides $q-1 . Z_{4} \times Z_{2}$ occurs if and only if $p=2$. $\quad Z_{3}$ occurs if and only if $\left[k^{*}:\left(k^{*}\right)^{3}\right] \geqq 9$ and $Z_{3} \times Z_{3}$ occurs if and only if $\left[k^{*}:\left(k^{*}\right)^{3}\right] \geqq 27 . \quad Z_{5}$ occurs if and only if $\left[k^{*}:\left(k^{*}\right)^{5}\right] \geqq$ 25 , i.e., $p=5$ or 5 divides $q-1$.

Arrange the simple roots in the diagram

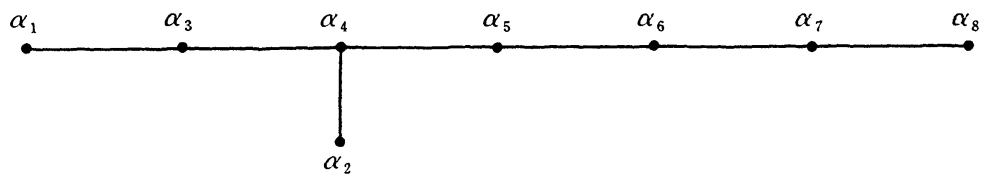

Letting $\beta=-\left({ }^{2454321}\right), \Phi$ contains a subsystem of type $D_{8}$ spanned by

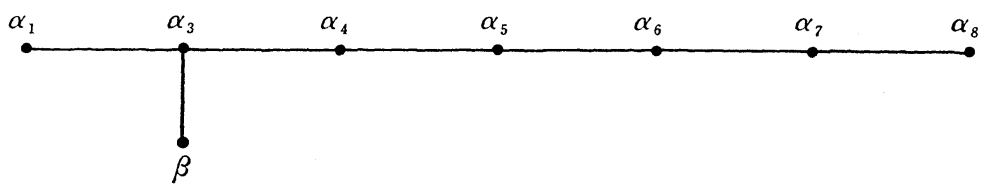

giving an inclusion of Weyl groups $W\left(D_{8}\right)<W\left(E_{8}\right)$. Comparing orders, $2^{14} \cdot 3^{2} \cdot 5 \cdot 7$ and $2^{14} \cdot 3^{5} \cdot 5^{2} \cdot 7$, we see that a 2-Sylow subgroup of $W\left(D_{8}\right)$ is also a 2-Sylow subgroup of $W\left(E_{8}\right)$. Thus we may assume that a 2-Sylow subgroup of $R$ is contained in $W\left(D_{8}\right)$; we first classify these groups.

In this realization, the orderings determined by the positive roots are not compatible between the $D_{8}$ and $E_{8}$ root systems. However, easy modifications of the proofs show that Lemmas 1 and 3 of $\S D_{n}$ hold. Adding the condition $w \alpha_{2} \equiv \alpha_{2}$ in $E_{8}$, we see that possible 
elements in $W\left(D_{8}\right) \cap R$, grouped by $W\left(E_{8}\right)$-conjugacy classes, are conjugate to

$$
\begin{aligned}
& 4 A_{1}: C_{5} C_{6} C_{7} C_{8},(12)(34)(56)(78) C_{7} C_{8}, \\
& 6 A_{1}: C_{3} C_{4} C_{5} C_{6} C_{7} C_{8},(12)(34) C_{5} C_{6} C_{7} C_{8}, \\
& 7 A_{1}:(12) C_{3} C_{4} C_{5} C_{6} C_{7} C_{8}, \\
& 8 A_{1}: w_{0}=-1, \\
& 2 D_{4}\left(a_{1}\right):(12)(34)(56)(78) C_{2} C_{4} C_{6} C_{7}, \\
& D_{4}\left(a_{1}\right)+3 A_{1}:(12)(34)(56) C_{4} C_{6} C_{7} C_{8}, \text { or } \\
& D_{4}\left(a_{1}\right)+4 A_{1}:(12)(34) C_{2} C_{4} C_{5} C_{6} C_{7} C_{8} .
\end{aligned}
$$

Suppose there is an element of order 4 in $R$. If there is one of type $2 D_{4}\left(a_{1}\right)$, we may assume it is (12) (34) (56) (78) $C_{2} C_{4} C_{6} C_{7}$. Then $\lambda$ is given by

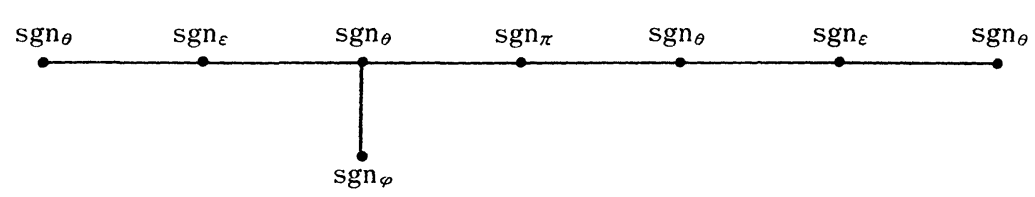

with $\left|\left\langle\operatorname{sgn}_{\theta}, \operatorname{sgn}_{\varphi}, \operatorname{sgn}_{\varepsilon}, \operatorname{sgn}_{\pi}\right\rangle\right|=16$. This can occur if and only if $\left[k^{*}:\left(k^{*}\right)^{2}\right] \geqq 16$, and in this case, $\Delta^{\prime}=\dot{\phi}$.

Then

$$
\begin{aligned}
R= & W_{\lambda} \\
= & \left\langle(12)(34)(56)(78) C_{7} C_{8},(13)(24)(57)(68) C_{6} C_{8},(15)(26)(37)(48) C_{4} C_{8}\right\rangle \\
& \ltimes\left\langle C_{1} C_{3} C_{5} C_{7}, C_{2} C_{4} C_{6} C_{8}, C_{1} C_{2} C_{3} C_{4}, C_{3} C_{4} C_{5} C_{6}\right\rangle
\end{aligned}
$$

is nonabelian of order 128 and has 65 conjugacy classes. $R$ odm $\left\langle w_{0}\right\rangle$ is abelian of order 64 .

Now suppose that $R$ does not contain an element of type $2 D_{4}\left(a_{1}\right)$, but does contain (12) (34) $(56) C_{4} C_{6} C_{7} C_{8}$ of type $D_{4}\left(a_{1}\right)+3 A_{1}$. Then $\lambda$ is given by

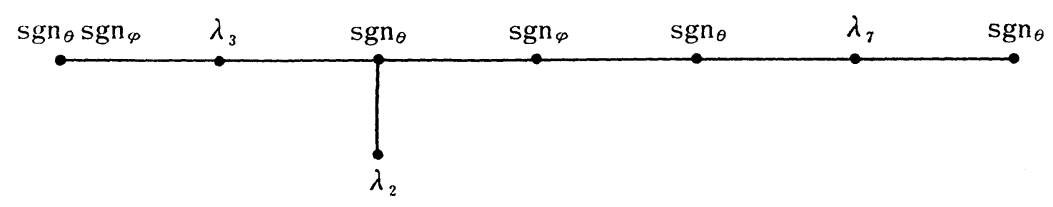

with $\lambda_{3}^{2}=\operatorname{sgn}_{\theta}$ and $\lambda_{2}^{2} \lambda_{7}^{2}=\operatorname{sgn}_{\theta} \operatorname{sgn}_{\varphi}$. This can occur if and only if $p=2$ or 4 divides $q-1$. An examination of other possible elements in $R$ with this one shows that $R \cong Z_{4}$ if $\lambda_{2}$ and $\lambda_{7}$ satisfy no additional conditions. If $\lambda_{7}$ has order $2, \lambda_{7} \notin\left\langle\operatorname{sgn}_{\theta}, \operatorname{sgn}_{\varphi}\right\rangle$, then $C_{1} C_{2} C_{3} C_{4} \in$ $R \cong \boldsymbol{Z}_{4} \times \boldsymbol{Z}_{2}$. If instead $\lambda_{7}^{2}=\operatorname{sgn}_{\varphi}, \lambda_{2} \lambda_{3}^{-1}$ has order $2, \lambda_{2} \lambda_{3}^{-1} \notin\left\langle\operatorname{sgn}_{\theta}, \operatorname{sgn}_{\varphi}\right\rangle$, then (12) (35) (46) (78) $C_{1} C_{2} \in R \cong \boldsymbol{Z}_{4} \times \boldsymbol{Z}_{2}$. These two cases occur if and only if $p=2$.

Next, assume that $R$ does not contain elements of types $2 D_{4}\left(a_{1}\right)$ 
or $D_{4}\left(a_{1}\right)+3 A_{1}$, but does contain (12) $(34) C_{2} C_{4} C_{5} C_{6} C_{7} C_{8}$ of type $D_{4}\left(a_{1}\right)+$ $4 A_{1}$. Then $\lambda$ is given by

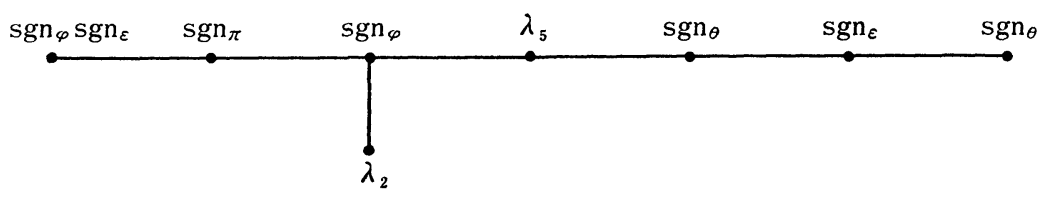

with $\lambda_{5}^{2}=\operatorname{sgn}_{\theta}$ and $\lambda_{2}^{2}=\operatorname{sgn}_{\theta} \operatorname{sgn}_{\varepsilon}$, and $R \cong Z_{4}$. This case occurs if and only if $\left[k^{*}:\left(k^{*}\right)^{2}\right] \geqq 16$.

Now assume that $R$ contains no elements of order 4 . An explicit list shows that a 2-Sylow subgroup of $R$ is then $\boldsymbol{Z}_{2}^{n}$ with $0 \leqq n \leqq 4$. Further, $\boldsymbol{Z}_{2}^{n}$ occurs if and only if $\left[k^{*}:\left(k^{*}\right)^{2}\right] \geqq 2^{n+1}, 0 \leqq n \leqq 4$.

Using the fact that no elements of order 6 can be in an $E_{8} R$ group, it is easy to see that the other $R$-groups which occur are isomorphic to $\boldsymbol{Z}_{3}, \boldsymbol{Z}_{3} \times \boldsymbol{Z}_{3}$ or $\boldsymbol{Z}_{5}$.

$R \cong Z_{3}$ may be generated by an element of type $3 A_{2}$ or $4 A_{2}$.

To construct $R \cong \boldsymbol{Z}_{3} \times \boldsymbol{Z}_{3}$, note that $\Phi$ contains a subsystem of type $\mathrm{A}_{8}$ spanned by $\left\{\alpha_{0}, \alpha_{1}, \alpha_{3}, \alpha_{4}, \alpha_{5}, \alpha_{6}, \alpha_{7}, \alpha_{8}\right\}$, where $\alpha_{0}={ }^{1354332}$. Letting $\alpha_{0}=e_{1}-e_{2}, \quad \alpha_{1}=e_{2}-e_{3}, \cdots, \quad \alpha_{8}=e_{8}-e_{9}, \quad R=\langle(123)$ (456) (789), (147) (258) (369)> occurs for the character

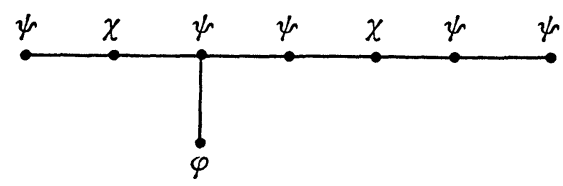

with $\psi, \chi$ and $\varphi$ of order 3 and $|\langle\psi, \chi, \varphi\rangle|=27$.

$\boldsymbol{Z}_{5}$ will occur as an $R$-group, generated by an element of type $2 A_{4}$, if and only if $\left[k^{*}:\left(k^{*}\right)^{5}\right] \geqq 25$.

8. Type $\mathrm{F}_{4} . \quad \Phi^{v}=\left\{ \pm 2 e_{k}, \pm e_{i} \pm e_{j}, \pm e_{1} \pm e_{2} \pm e_{3} \pm e_{4} \mid 1 \leqq k \leqq 4\right.$, $1 \leqq i<j \leqq 4\}$ is of type $\mathrm{F}_{4}$. A base for $\Phi^{v}$ is given by $\alpha_{1}=e_{2}-e_{3}$, $\alpha_{2}=e_{3}-e_{4}, \alpha_{3}=2 e_{4}$, and $\alpha_{4}=e_{1}-e_{2}-e_{3}-e_{4}$.

$\Phi^{\prime}=\left\{ \pm e_{i} \pm e_{j} \mid 1 \leqq i<j \leqq 4\right\}$ forms a sub-root system of type $D_{4}$ with Weyl group $W\left(\Phi^{\prime}\right) \cong S_{4} \ltimes \boldsymbol{Z}_{2}^{3}$ acting as permutations and even sign changes on the $e_{i}$. The Weyl group for $\Phi$ and $\Phi^{v}$ of type " $\mathrm{F}_{4}$ is $S_{3} \ltimes W\left(\Phi^{\prime}\right) \cong S_{3} \ltimes\left(S_{4} \ltimes Z_{2}^{3}\right)$, where $S_{3}$ acts as permutations of $e_{1}-e_{2}, e_{3}-e_{4}$, and $e_{3}+e_{4}$.

If $w_{\beta} \alpha=\alpha-n(\alpha, \beta) \beta$, the Cartan matrix $[n(\alpha, \beta)]$ of $\Phi^{v}$ is

$$
\left[\begin{array}{rrrr}
2 & -1 & 0 & 0 \\
-1 & 2 & -1 & 0 \\
0 & -2 & 2 & -1 \\
0 & 0 & -1 & 2
\end{array}\right] \text {. }
$$


The reader is referred to Bourbaki [2] for more details.

THEOREM $\mathrm{F}_{4} . \quad R \cong 1, Z_{2}$ or $\boldsymbol{Z}_{3} . \quad Z_{3}$ can occur as an $R$-group if and only if $p=3$ or 3 divides $q-1$.

Lemma 1. Suppose $w=s d \in R$ with $s \in S_{3}$ and $d \in S_{4} \ltimes Z_{2}^{3}$. Then $s$ has order 1 or 3.

Proof. $s \in S_{3}$ has order 1,2 , or 3 , so that $w=s d, w^{2}$, or $w^{3}$ is in the normal subgroup $S_{4} \ltimes Z_{2}^{3}$. Further, this element must be able to give reducibility for $D_{4}$, so that $w, w^{2}$, or $w^{3}$ is conjugate to one of $1, c_{3} c_{4}, c_{1} c_{2} c_{3} c_{4},(12) c_{3} c_{4},(12)(34)$, or (12) (34) $c_{2} c_{4}$.

But of these, only $1, c_{1} c_{2} c_{3} c_{4}$, and $(12) c_{3} c_{4}$ can be in an $R$-group for $\Phi$ of type $\mathrm{F}_{4}$. Thus $w, w^{2}$, or $w^{3}$ is conjugate to one of $1, c_{1} c_{2} c_{3} c_{4}$, or $(12) c_{3} c_{4}$.

Suppose that $s$ has order 2 , so that $w^{2}=1, c_{1} c_{2} c_{3} c_{4}$, or $(12) c_{3} c_{4}$. We may assume that $s=w_{\alpha_{3}}=c_{4}=\left(e_{3}-e_{4}, e_{3}+e_{4}\right)$. Then if $d=\sigma c$ with $\sigma \in S_{4}$ and $c \in Z_{2}^{3}, w^{2}=c_{4}\left(\sigma c c_{4} \sigma^{-1}\right)\left(\sigma^{2} c \sigma^{-2}\right) \sigma^{2}$. Since $(12) \neq \sigma^{2}$ for any $\sigma$, we must have $\sigma^{2}=1$ and thus $c c_{4}\left(\sigma c c_{4} \sigma^{-1}\right)=w^{2}=1$ or $c_{1} c_{2} c_{3} c_{4}$.

By conjugation we may assume that $\sigma=1$, (12), (34), or (12) (34). But then $w^{2} \neq c_{1} c_{2} c_{3} c_{4}$ for any $c \in Z_{2}^{3}$, so we have $w^{2}=1$. But $\sigma=$ (12) (34) will not give $w^{2}=1$ for any $c$.

Thus $\sigma=1$ and $w=c_{4} c, c \in Z_{2}^{3}$, or $\sigma=(12)$ and $c=1, c_{1} c_{2}, c_{3} c_{4}$, or $c_{1} c_{2} c_{3} c_{4}$, or $\sigma=(34)$ and $c$ is conjugate to $c_{2} c_{3}$ or $c_{2} c_{4}$. Then $w=\sigma c_{4} c$ is conjugate to one of $c_{4}, c_{2} c_{3} c_{4}$, or (12) $c_{4}$. But none of these can be in an $R$-group for $\Phi$ of type $F_{4}$. Thus $s$ can not have order 2.

If $s=1$, then $w=s d \in W\left(\Phi^{\prime}\right)$.

Lemma 2. Suppose $R \leqq W\left(\Phi^{\prime}\right)$. Then $R \cong Z_{2}$.

Proof. Any element of $R \cap W\left(\Phi^{\prime}\right)$ is conjugate to one of 1 , $c_{1} c_{2} c_{3} c_{4}$, or $(12) c_{3} c_{4} . \quad c_{1} c_{2} c_{3} c_{4}$ can not be in an $R$-group with any conjugate of (12) $c_{3} c_{4}$, so if $c_{1} c_{2} c_{3} c_{4} \in R$, then $R=\left\langle c_{1} c_{2} c_{3} c_{4}\right\rangle \cong Z_{2}$.

Suppose $(12) c_{3} c_{4} \in R$. Then $\lambda$ is given by

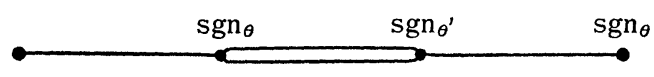

where $\operatorname{sgn}_{\theta} \neq \operatorname{sgn}_{\theta^{\prime}}$ are of order 2. If there is another nontrivial element of $R$, we may assume by conjugation that it is $c_{1} c_{2}(34)$. We then would need $\alpha_{1}$ to correspond to a character $\Psi$ with $\Psi^{2}=$ $\operatorname{sgn}_{\theta} \operatorname{sgn}_{\theta^{\prime}}$. But then $2 \alpha_{1}+2 \alpha_{2}+\alpha_{3}+\alpha_{4} \in \mathscr{L} \cap \Phi^{v}$ and $c_{1} c_{2}(34)$ sends this to a negative root, so it is not in an $R$-group with (12) $c_{3} c_{4}$. Thus $R=\left\langle(12) c_{3} c_{4}\right\rangle \cong Z_{2}$.

Now suppose there exists an element $w=s d \in R$ with $s \neq 1$, 
$s \in S_{3}$ and $d \in W\left(\Phi^{\prime}\right)$. Then $s$ has order 3 by Lemma 1 , and $w^{3} \in W\left(\Phi^{\prime}\right)$ must be conjugate to $1, c_{1} c_{2} c_{3} c_{4}$, or (12) $c_{3} c_{4}$. Thus $w$ has order 3 or 6 .

Consider the elements $w_{\alpha_{3}} w_{\alpha_{4}}, w_{\alpha_{1}} w_{\alpha_{2}},\left(w_{\alpha_{2}} w_{\alpha_{3}} w_{\alpha_{4}}\right)^{2}=w_{2 \alpha_{2}+\alpha_{3}} w_{\alpha_{3}+\alpha_{4}}$, and $\left(w_{\alpha_{1}} w_{\alpha_{2}} w_{\alpha_{3}} w_{\alpha_{4}}\right)^{2}$ of order 3 . The first 3 elements can not give reducibility. The last gives reducibility if $\lambda$ is given by

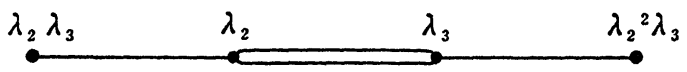

where $\lambda_{2} \neq \lambda_{3}^{ \pm 1}$ are characters of order 3 .

The above 4 elements are pairwise nonconjugate. Further, none is conjugate to the inverse of another. Since the order of the Weyl group $W$ is $3^{2} \cdot 2^{7}$, we see that any 3-Sylow subgroup of $W$ consists of 1 and conjugates of the above four elements and their inverses. Thus there is a unique subgroup of order 3 in any 3-Sylow subgroup which can be part of an $R$-group.

Thus any element of order 3 in $R$ is conjugate to $\left(w_{\alpha_{1}} w_{\alpha_{2}} w_{\alpha_{3}} w_{\alpha_{4}}\right)^{4}$ or its inverse. In this case all $\alpha$ correspond to characters of order 3 , and thus $R$ can not contain an element of order 2 , which would have to be conjugate to $c_{1} c_{2} c_{3} c_{4}$ or (12) $c_{3} c_{4}$. Thus an element of order 6 can not occur, and we have shown that $R \cong\{1\}, Z_{2}$, or $Z_{3}$.

Explicitly, if $R \neq\{1\}$, then $R$ is conjugate to one of $\left\langle c_{1} c_{2} c_{3} c_{4}\right\rangle \cong Z_{2}$ with all $\lambda_{\alpha}$ of order 2 , or $\left\langle(12) c_{3} c_{4}\right\rangle \cong Z_{3}$ with $\lambda$ given by

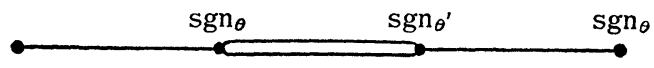

with $\operatorname{sgn}_{\theta} \neq \operatorname{sgn}_{\theta^{\prime}}$, or $\left\langle\left(w_{\alpha_{1}} w_{\alpha_{2}} w_{\alpha_{3}} w_{\alpha_{4}}\right)^{4}\right\rangle \cong Z_{3}$ with $\lambda$ given by

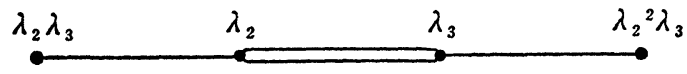

where $\lambda_{2} \neq \lambda_{3}^{ \pm 1}$ are of order 3 .

We note that if $=\boldsymbol{R}$, then $R=\{1\}$, and thus $\operatorname{Ind}_{B}^{G} \lambda$ is irreducible if $G$ is a Chevalley group of type $F_{4}$ over the reals.

9. Type $\mathrm{G}_{2}$. Let $\{\alpha, \beta\}$ be a base for $\Phi^{v}$ with Cartan matrix $\left[\begin{array}{rr}2 & -1 \\ -3 & 2\end{array}\right]$. The positive roots in $\Phi^{v}$ are $\alpha, \beta, \alpha+\beta, 2 \alpha+\beta, 3 \alpha+\beta$, and $3 \alpha+2 \beta$. The Weyl group $W$ is a dihedral group of order 12 .

THEOREM $\mathrm{G}_{2} . \quad R=\{1\}$ or $R=\left\langle w_{0}\right\rangle \cong Z_{2}$, where $w_{0}$ is the Weyl group element of maximal length.

One checks that the element $w_{0}$ of maximal length is the only Weyl group element giving reducibility. $R=\left\langle w_{0}\right\rangle$ if and only if $\alpha$ and $\beta$ correspond to distinct characters of order 2 .

If $\mathfrak{l}$ is nonArchimedean, $\left[\mathfrak{f}^{*}:\left(\mathfrak{l}^{*}\right)^{2}\right] \geqq 4$, and such characters exist. If $\mathfrak{l}=R$, then $R=\{1\}$ and $\operatorname{Ind}_{B}^{G} \lambda$ will be irreducible. 


\section{CHAPTER III \\ ON THE DECOMPOSITION OF $\operatorname{Ind}_{B}^{a} \lambda$}

1. Multiplicities of the irreducible components. If $R$ is abelian, then there are $|R|$ irreducible components, each occuring with multiplicity 1.

Write $\boldsymbol{C}[R]=M_{m_{1}}(\boldsymbol{C}) \oplus \cdots \oplus M_{m_{k}}(\boldsymbol{C})$. Then $m_{1}, m_{2}, \cdots, m_{k}$ are the multiplicities of the $k$ inequivalent irreducible components of $\operatorname{Ind}_{B}^{G} \lambda . \quad k$ is equal to the dimension of the center of $C[R]$, which equals the number of conjugacy classes in $R$. Further, the $m_{i}$ are the degress of the irreducible representations of the group $R$. We note that if $R$ has a normal abelian subgroup $R^{\prime}$, then the degrees $m_{i}$ divide the index of $R^{\prime}$ in $\boldsymbol{R}$, by Ito's Theorem.

Suppose $R$ is non-abelian. Then $G$ is of type $\mathrm{D}_{n}, \mathrm{E}_{7}$ or $\mathrm{E}_{8}$. Suppose $G$ is type $\mathrm{D}_{n}$, with $n$ odd. Then $R \cong Z_{4} \times R^{\prime \prime}$ contains a normal abelian subgroop $R^{\prime}$ of index 2 , so $m_{i}=1$ or 2 . If $p$ is odd, then $R \cong \boldsymbol{Z}_{4} \ltimes\left(\boldsymbol{Z}_{2} \times \boldsymbol{Z}_{2}\right)$ has order 16 and there are 10 conjugacy classes in $R$. Thus we have the decomposition $16=2 \cdot 2^{2}+8 \cdot 1^{2}$, and $\operatorname{Ind}_{B}^{G} \lambda$ decomposes into 2 irreducible components of multiplicity 2 , and 8 irreducible components of multiplicity 1 .

If $p=2$, there may be more factors of $Z_{2}$ in $R$. We note that $R \cong \boldsymbol{Z}_{4} \ltimes\left(\boldsymbol{Z}_{2}^{4}\right)$ has 28 conjugacy classes, giving the decomposition $64=12 \cdot 2^{2}+16 \cdot 1^{2}$, and $R \cong Z_{4} \ltimes\left(Z_{2}^{6}\right)$ has 88 conjugacy classes, giving the decomposition $256=56 \cdot 2^{2}+32 \cdot 1^{2}$.

Suppose $G$ is type $\mathrm{D}_{n}$ with $n$ even. Then any non-abelian $R$ is isomorphic to $\left(\boldsymbol{Z}_{2} \times \cdots \times \boldsymbol{Z}_{2}\right) \ltimes R^{\prime}$. If $p$ is odd, $R^{\prime}$ is the group of even sign changes on $\left\{e_{n}, e_{n-1}, e_{n-2}, e_{n-3}\right\}$ and the first factor is $\langle(12)(34) \cdots(n-1 n)\rangle$ or $\langle(12)(34) \cdots(n-1 n),(13)(24) \cdots(n-2 n)\rangle$.

In the first case, $m_{i}=1$ or $2,|R|=16$ and there are 10 conjugacy classes in $R .16=2 \cdot 2^{2}+8 \cdot 1^{2}$ gives the decomposition into 2 irreducible components of multiplicity 2 , and 8 of multiplicity 1.

In the second case, $|R|=32$ and there are 17 conjugacy classes. The two possible decompositions are $32=4^{2}+16 \cdot 1^{2}=5 \cdot 2^{2}+12 \cdot 1^{2}$. But since $R /\left\langle c_{n} c_{n-1} c_{n-2} c_{n-3}\right\rangle$ is abelian, there are at least 16 one-dimensional representations of $R$, so the decomposition must be $32=4^{2}+$ $16 \cdot 1^{2}$. Thus $\operatorname{Ind}_{B}^{G} \lambda$ decomposes into 16 irreducible components of multiplicity 1 , and 1 component of multiplicity 4 .

If $G$ is type $\mathrm{E}_{7}$, the nonabelian $R$-groups are the dihedral group $D$ of order 8 and $D \times Z_{2} . \quad R \cong D$ gives the decomposition $8=1 \cdot 2^{2}+4 \cdot 1^{2}$ and $R \cong D \times Z_{2}$ gives the decomposition $16=2 \cdot 2^{2}+8 \cdot 1^{2}$.

If $G$ is type $\mathrm{E}_{8}$, the nonabelian $R$-group has order 128,65 conjugacy classes, and $R /\left\langle w_{0}\right\rangle$ is abelian of order 64 . This gives the decomposition $128=1 \cdot 8^{2}+64 \cdot 1^{2}$, so $\operatorname{Ind}_{B}^{G} \lambda$ decomposes into 1 irre- 
ducible components with multiplicity 8 , and 64 irreducible components each with multiplicity 1.

2. Some analysis on $L^{2}(V)$. In this section we realize the operators $a(w, \lambda)$ on $L^{2}(V)$ via a Fourier transform, where $V$ is the unipotent radical of the Borel subgroup opposed to $B$. We find a class of functions in $L^{2}(V)$ on which $\hat{a}(w, \lambda)$ acts as multiplication by a bounded function $M(w, \lambda)$. This class has nonzero intersection with each invariant subspace for groups of type $\mathrm{A}_{n}$ and $\mathrm{B}_{n}$.

Write $\varphi_{\hat{o}}(y)$ for $\varphi_{\hat{o}}\left(\begin{array}{ll}1 & y \\ 0 & 1\end{array}\right)$ in $U_{\delta}$ and let $n_{\alpha}=\varphi_{\alpha}\left(\begin{array}{rr}0 & 1 \\ -1 & 0\end{array}\right)$ for $\alpha$ simple, where $\varphi_{\delta}: \mathrm{SL}(2) \rightarrow G$ is the canonical homomorphism corresponding to the root $\delta$.

Write $V=\prod_{\delta<0} U_{\delta}$ in some fixed order. Since each $U_{\delta}$ is isomorphic to $k$, this gives a topological isomorphism of $V$ with the product of $\left|\Phi^{-}\right|$copies of $\mathfrak{k}$. We then define a Fourier transform on $L^{2}(V)$ by $\hat{f}\left(\Pi_{\delta<0} \varphi_{\delta}\left(c_{\delta}\right)\right)=\int f\left(\Pi_{\delta<0} \varphi_{\delta}\left(y_{\delta}\right)\right) \bar{\chi}\left(\sum_{\delta<0} c_{\hat{\delta}} y_{\delta}\right) \Pi d y_{\hat{\delta}}$, where $\chi$ is a fixed additive character of $\mathfrak{t}$ with conductor the ring of integers.

Fix a simple root $\alpha>0$. Then

$$
\begin{aligned}
A\left(w_{\alpha}, \lambda\right) f(g) & =A\left(n_{\alpha}, \lambda\right) f(g) \\
& =\int_{U_{\alpha}} f\left(g \varphi_{\alpha}\left(\left(\begin{array}{ll}
1 & u \\
0 & 1
\end{array}\right)\left(\begin{array}{rr}
0 & 1 \\
-1 & 0
\end{array}\right)\right)\right) d u \\
& =\int f\left(g \varphi_{\alpha}\left(\begin{array}{cc}
1 & 0 \\
1 / u & 1
\end{array}\right) \varphi_{\alpha}\left(\begin{array}{cc}
-u & 1 \\
0 & -1 / u
\end{array}\right)\right) d u \\
& =\int f\left(g \varphi_{\alpha}\left(\begin{array}{cc}
1 & 0 \\
1 / u & 1
\end{array}\right)\right) \lambda_{\alpha}^{-1}(-u) \frac{d u}{|u|} \\
& =\int f\left(g \varphi_{\alpha}\left(\begin{array}{ll}
1 & 0 \\
u & 1
\end{array}\right)\right) \lambda_{\alpha}(-u) \frac{d u}{|u|} .
\end{aligned}
$$

Let $g \in V, g=\prod_{\tilde{\delta}<0} \varphi_{\tilde{\delta}}\left(y_{\tilde{\delta}}\right)$. Then

$$
g \varphi_{\alpha}\left(\begin{array}{ll}
1 & 0 \\
u & 1
\end{array}\right)=\prod \varphi_{\delta}\left(y_{\delta}\right) \cdot \varphi_{-\alpha}(u)=\prod_{\delta<0} \varphi_{\delta}\left(y_{\delta}+P_{\delta}\left(y_{\beta}, u\right)\right),
$$

where the $P_{\delta}=P_{\delta, \alpha}$ are polynomials arising from Chevalley's commutation relations. Make the change of variables $y_{-\alpha} \mapsto y_{-\alpha}-u$ to define polynomials $Q_{\delta}\left(y_{\beta}, u\right)$. Then $Q_{\delta} \equiv 0$ if $\delta$ is simple.

Consider the operator $A\left(w_{\alpha}, \lambda\right)$ under the Fourier transform. Let $\hat{g}=\prod \varphi_{\hat{o}}\left(c_{\delta}\right)$. Then

$$
\hat{A}\left(w_{\alpha}, \lambda\right) \hat{f}(\hat{g})=\iint f\left(\Pi \varphi_{\delta}\left(y_{\dot{\delta}}+P\left(y_{\beta}, u\right)\right)\right) \lambda_{\alpha}(-u) \frac{d u}{|u|} \bar{\chi}\left(\sum c_{\dot{\delta}} y_{\delta}\right) \Pi d y_{\dot{o}}
$$




$$
\begin{aligned}
= & \iint f\left(\Pi \varphi_{\delta}\left(y_{\delta}+Q_{\delta}\left(y_{\beta}, u\right)\right)\right) \lambda_{\alpha}(-u) \bar{\chi}\left(-c_{-\alpha} u\right) \frac{d u}{|u|} \\
& \times \bar{\chi}\left(\sum c_{\delta} y_{\delta}\right) \Pi d y_{\delta} .
\end{aligned}
$$

We define a function $f \in C_{c}^{\infty}(V)$ as follows. For $\delta<0, \delta$ simple, let $\hat{f}_{\delta}$ be any function in $C_{c}^{\infty}\left(\mathfrak{k}^{*}\right)$, i.e., such that the support of $\hat{f}_{\delta}$ avoids zero. Let $S_{\delta}$ be the support of $f_{\hat{\delta}}$. If $\delta<0$ is nonsimple with $Q_{\delta} \equiv 0$, take any $f_{\delta} \in C_{c}^{\infty}\left(U_{\delta}\right)$, and let $S_{\delta}$ be its support.

Define the other $S_{\delta}$ inductively from right to left in the product $\Pi_{\tilde{\delta}<0} U_{\tilde{\delta}}$. If $S_{\beta}$ is defined for all $\beta$ to the right of $\delta$ in the product, let $S_{\delta}$ be the fractional ideal generated by $\left\{Q_{\delta, \alpha}\left(y_{\beta}, u c_{-\alpha}^{-1}\right) \mid y_{\beta} \in S_{\beta}\right.$, $u \in \mathscr{O}$ if $\lambda_{\alpha}$ is unramified and $|u|=q^{h}$ if $\lambda_{\alpha}$ is ramified of degree $h$, and $c_{-\alpha} \in \operatorname{supp} \hat{f}_{-\alpha}$ for $\alpha$ simple\}. Define $f_{\delta}$ to be the characteristic function of $S_{\dot{\delta}}$.

For root systems of type $\mathrm{A}_{n}, \mathrm{~B}_{n}, \mathrm{C}_{n}, \mathrm{D}_{n}$ and $\mathrm{G}_{2}$, we may arrange the negative roots such that $Q_{\delta, \alpha}\left(y_{\beta}, u\right) \not \equiv$ implies $Q_{\beta, \alpha} \equiv 0, Q_{\delta, \alpha}\left(y_{\beta}, u\right) \not 0$ implies $\beta \neq-\alpha$, and $Q_{\delta, \alpha}\left(y_{\beta}, u\right)=Q_{\delta, \alpha}\left(y_{\beta}\right) u$. Then if $f=\prod f_{\delta}$,

$$
\begin{aligned}
& \hat{A}\left(w_{\alpha}, \lambda\right) \hat{f}(\hat{g})=\iiint_{\substack{\beta \\
Q_{\beta} \equiv 0}} f_{\beta}\left(y_{\beta}\right) \bar{\chi}\left(\sum c_{\beta} y_{\beta}\right) \prod_{\substack{\delta \\
Q_{\delta} \neq 0}} f_{\delta}\left(y_{\delta}+Q_{\delta}\left(y_{\beta}\right) u\right) \bar{\chi}\left(\sum c_{\delta} y_{\delta}\right) \\
& \times \lambda_{\alpha}(-u) \bar{\chi}\left(-c_{-\alpha} u\right) \frac{d u}{|u|} \Pi d y_{\delta} \Pi d y_{\beta}=0
\end{aligned}
$$

unless $y_{\beta} \in S_{\beta}$ for all $\beta$ with $Q_{\beta} \equiv 0$. Fix $y_{\beta} \in S_{\beta}$ and consider

$$
\iint \prod_{Q_{\delta} \neq 0} f_{\delta}\left(y_{\delta}-Q_{\delta}\left(y_{\beta}\right) u\right) \bar{\chi}\left(\sum c_{\delta} y_{\delta}\right) \lambda_{\alpha}(u) \bar{\chi}\left(c_{-\alpha} u\right) \frac{d u}{|u|} \Pi d y_{\delta} .
$$

This will be zero unless $y_{\delta}-Q_{\delta}\left(y_{\beta}\right) u \in S_{\delta}$ for all $\delta$. Thus we need only integrate $u$ over the intersection $\bigcap_{\delta}\left(1 / Q_{\delta}\left(y_{\beta}\right)\right)\left(y_{\delta}-S_{\delta}\right)=$ $\left(1 / Q_{\delta_{0}}\left(y_{\beta}\right)\left(y_{\delta_{0}}-S_{\delta_{0}}\right)\right.$, for some $\delta_{0}$, and integrate $y_{\delta}$ over the coset

Write

$$
\frac{Q_{\delta}\left(y_{\beta}\right)}{Q_{\delta_{0}}\left(y_{\beta}\right)} y_{\delta_{0}}+S_{\delta}
$$

$$
\int_{u_{\delta_{0}}}=\int_{s_{\delta_{0}}}+\sum \int_{s}
$$

where the sum is over shells $\mathscr{S}$ consisting of nonzero cosets of $S_{\tilde{o}_{0}}$. The above integral becomes

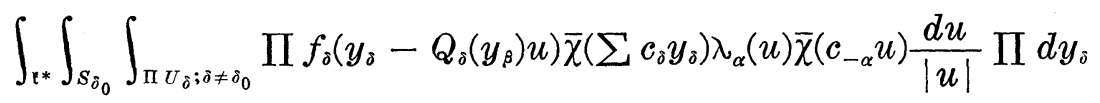

$$
\begin{aligned}
& +\sum_{\mathscr{S}} \iiint_{\mathscr{\mathcal { S }}} \Pi f_{\hat{o}}\left(y_{\hat{o}}-Q_{\hat{\delta}}\left(y_{\beta}\right) u\right) \bar{\chi}\left(\sum c_{\delta} y_{\hat{\delta}}\right) \lambda_{\alpha}(u) \bar{\chi}\left(c_{-\alpha} u\right) \frac{d u}{|u|} \Pi d y_{\hat{o}} .
\end{aligned}
$$

In each term in the sum, we are integrating $\lambda_{\alpha}(u) \bar{\chi}\left(c_{-\alpha} u\right)$ over 
a shell $\left(1 / Q_{\delta_{0}}\left(y_{\beta}\right)\right) \mathscr{S}$ which is disjoint from $S_{\delta_{0}} / Q_{\delta_{0}}\left(y_{\beta}\right)$. Thus we are integrating $\lambda_{\alpha}(u) \bar{\chi}(u)$ over a shell disjoint from $\left(1 / Q_{\delta_{0}}\left(y_{\beta}\right)\right) c_{-\alpha} S_{\delta_{0}}$, which gives zero, by the definition of $S_{\delta_{0}}$ and properties of the gamma function $[29,35]$.

We are left with only the first term. Note that

$$
\int_{\left(1 / Q_{\tilde{\delta}_{0}}\left(y_{\beta}\right)\right) S_{\tilde{\delta}_{0}}} \lambda_{\alpha}(u) \bar{\chi}\left(c_{-\alpha} u\right) \frac{d u}{|u|}=\lambda_{\alpha}^{-1}\left(c_{-\alpha}\right) \cdot \Gamma\left(\lambda_{\alpha}\right) .
$$

We get that

$$
\begin{aligned}
\hat{A}\left(w_{\alpha} \lambda\right) \hat{f}(\hat{g})= & \int_{\Pi 1 U_{\delta} ; \dot{\hat{o}} \neq \delta_{0}} \int_{S_{\delta_{0}}} \int_{t^{*}} \Pi f_{\beta}\left(y_{\beta}\right) \bar{\chi}\left(c_{\beta} y_{\beta}\right) \Pi f_{\delta}\left(y_{\delta}-Q_{\delta}\left(y_{\beta}\right) u\right) \\
& \times \bar{\chi}\left(\sum c_{\delta} y_{\delta}\right) \lambda_{\alpha}(u) \bar{\chi}\left(c_{-\alpha} u\right) \frac{d u}{|u|} \Pi d y_{\delta} \Pi d y_{\beta} .
\end{aligned}
$$

This is zero unless $y_{\beta} \in S_{\beta}, c_{-\alpha} \in \operatorname{supp} \hat{f}_{-\alpha}$ and $u \in 1 / c_{-\alpha} \times\left(\mathfrak{p}^{-h} \mid \mathfrak{p}^{-h+1}\right)$ for $\lambda_{\alpha}$ ramified of degree $h$, or $u \in 1 / c_{-\alpha} \times \mathscr{O}$ for $\lambda_{\alpha}$ unramified. But then $Q_{\hat{\delta}}\left(y_{\beta}\right) u \in S_{\hat{\delta}}$ and $f\left(y_{\delta}-Q_{\delta}\left(y_{\beta}\right) u\right)=f\left(y_{\delta}\right)$. Thus for such $\hat{f}$,

$$
\hat{A}\left(w_{\alpha}, \lambda\right) \hat{f}(\hat{g})=\lambda_{\alpha}^{-1}\left(c_{-\alpha}\right) \Gamma\left(\lambda_{\alpha}\right) \prod_{\hat{\delta}<0} \hat{f}_{\hat{\delta}}\left(c_{\delta}\right)=\lambda_{\alpha}^{-1}\left(c_{-\alpha}\right) \Gamma\left(\lambda_{\alpha}\right) \hat{f}(\hat{g}) .
$$

Thus $\hat{a}\left(w_{\alpha}, \lambda\right)=\left(1 / \Gamma\left(\lambda_{\alpha}\right)\right) A\left(w_{\alpha}, \lambda\right)$ acts on such $\hat{f}$ as multiplication by $M\left(w_{\alpha}, \lambda\right)=\lambda_{\alpha}^{-1}\left(c_{-\alpha}\right)$. Then if $w=w_{\alpha_{1}} w_{\alpha_{2}} \cdots w_{\alpha_{l}}, \hat{\mathrm{a}}(w, \lambda)$ acts on such $\hat{f}$ as multiplication by the function $M(w, \lambda)=M\left(w_{\alpha_{1}}, w_{\alpha_{2}} \cdots w_{\alpha_{l}} \lambda\right) \cdots$ $M\left(w_{\alpha l}, \lambda\right)$, by the cocycle condition.

We note that $w \mapsto M(w, \lambda)$ is a homomorphism, and further, that we may evaluate $M(w, \lambda)$ at $V_{-\alpha}$ for any simple root $\alpha$ to obtain a homomorphism from $W_{\lambda}$ into $\left(\mathfrak{t}^{*}\right)^{\wedge}$. If this homomorphism is injective on $R$ for some $\alpha$, then the linear independence of distinct characters of $\mathfrak{k}^{*}$ implies that the operators $\{a(w, \lambda) \mid w \in R\}$ are linearly independent. Further, we may write $|R|$ nonzero projections giving $\hat{f}$ as above in each invariant subspace.

The homomorphism is injective on $R$ for groups of type $\mathrm{A}_{n}$ and $\mathrm{B}_{n}$, but is not necessarily injective for groups of type $\mathrm{C}_{n}$ and $\mathrm{D}_{n}$. We may show the linear independence of the operators $\{a(w, \lambda) \mid w \in R\}$ for these groups as follows.

As in [37], let $f_{I}=f_{I, \lambda}$ be the function in $H_{\lambda}$ whose restriction to $K$ is supported on the Iwahori $I$ and is constant on $I \cap V$. Then $\mathfrak{a}(w, \lambda) f_{I}\left(w^{\prime}\right)=0$ if and only if $w w^{\prime} \neq 1$, provided that $l\left(w^{\prime}\right) \geqq l(w)$, that $\Gamma_{w}(\lambda)$ and $\Gamma_{w}(\lambda)$ are defined, and the characters $\lambda_{\beta}$ are ramified for all $\beta \in R(w)$. (The proof is by induction on the length of $w$. Write $w=w_{\alpha} \bar{w}$ with $l(\bar{w})=l(w)-1$ and use the fact that $\lambda_{\bar{w}-1_{\alpha}}$ is ramified.)

To show that $\{\mathfrak{a}(w, \lambda) \mid w \in R\}$ are linearly independent, it is enough to find a $w_{0} \in \boldsymbol{R}$ such that $\mathfrak{a}(w, \lambda) f_{I}\left(w_{0}\right)=0$ if and only if 
$w w_{0} \neq 1$. If all $\lambda_{\alpha}$ are ramified, use the above. Otherwise, since we know what groups $R$ can occur, we may check that $w_{0} \in R$ consisting of as many sign changes as possible will work for groups of type $\mathrm{C}_{n}$ and $\mathrm{D}_{n}$.

\section{REFERENCES}

1. A. Borel, Linear Algebraic Groups, Benjamin, New York, 1969.

2. N. Bourbaki, Groupes et algebras de Lie, Chap. IV, V et VI, Harmann, Paris, 1968.

3. F. Bruhat, Sur les representations induites des groups de Lie, Bull. Soc. Math. France, 84 (1956), 97-205.

4. F. Bruhat et J. Tits, Groupes reductifs sur un corps local, Paris, Institut des Hautes Etudes Scientifiques, Publ. Math., 41 (1972), 5-252.

5. R. Carter, Conjugacy classes in the Weyl group, Compositio Math., 25 (1972), 1-59.

6. W. Casselman, Some general results in the theory of admissible representations of p-adic reductive groups, preprint.

7. I. Gelfand, M. Graev and I. Pyattskii-Shapiro, Representation Theory and Automorphic Functions, Saunder, Philadelphia, 1969.

8. Harish-Chandra, On the theory of the Eisenstein integral, in Conference on Harmonic Analysis, Lecture Notes in Mathematics, 266, Springer-Verlag, New York, (1972), 123-149.

9. - Harmonic analysis on real reductive groups II, Wave-packets in the Schwartz space, Inv. Math. 36 (1976), 1-55.

10. - Harmonic analysis on real reductive III, The Maass-Selberg relations and the Plancherel formula, Ann. Math., 104 (1976), 117-201.

11. Harmonic analysis on reductive p-adic groups, in Harmonic Analysis on Homogeneous Spaces, Proc. Symposia Pure Math., Amer. Math. Soc., Providence, R.I., (1973), 167-192.

12. R. Howe and A. Silberger, Why any unitary principal series representation of $S L_{n}$ over a p-adic field decomposes simply, Bull. Amer. Math. Soc., 81 (1975), 599-601.

13. H. Jacquet, Representations des groupes linearies $p$-adiques, in Theory of Group Representations and Fourier Analysis, C.I.M.E., Montecatini, (1970), 121-220.

14. A. Knapp, Determination of intertwining operators, in Harmonic Analysis on Homogeneous Spaces, Proc. Symposia in Pure Math. Amer. Math. Soc., Providence, R.I., (1973), 263-268.

15. Commutativity of intertwining operators, Bull. Amer. Math. Soc., 79 (1973), 1016-1018.

16. Commutativity of intertwining operators II, Bull. Amer. Math. Soc., 82 (1976), 271-273.

17. A. Knapp and E. Stein, Intertwining operators for $S L(\mathrm{n}, R)$, preprint.

18. - Intertwining operators for semisimple groups, Ann. Math., 93 (1971), 489-578.

19. - Irreducibility theorems for the principal series, in Conference on Harmonic Analysis, Lecture Notes in Mathematics, 266, Springer-Verlag, New York, (1972), 197-214.

20. - Singular integrals and the principal series II, Proc. Nat. Acad. Sci., 66 (1970), 13-17.

21. - Singular integrals and the principal series III, Proc. Nat. Acad. Sci., 71 (1974), 4622-4624.

22. - Singular integrals and the principal series IV, Proc. Nat. Acad. Sci., 72 (1975), 2459-2461.

23. A. Knapp and G. Zuckerman, Classification of irreducible tempered representations of semisimple Lie groups, Proc. Nat. Acad. Sci., 73 (1976), 2178-2180. 
24. R. Kunze and E. Stein, Uniformly bounded representations and harmonic analysis of the $2 \times 2$ real unimodular group, Amer. J. Math., 82 (1960), 1-62.

25. - Uniformly bounded representations $I I$, analytic continuation of the principal series of the $n \times n$ complex unimodular group, Amer. J. Math., 83 (1961), $723-786$.

26. - Uniformly bounded representations III, intertwining operators for the principal series on semi-simple groups, Amer. J. Math., 89 (1967), 383-442.

27. I. MacDonald, Spherical functions on a group of p-adic type, Ramanujan Institute, University of Madras, Madras, India, 1972.

28. P. J. Sally, Jr., Unitary and uniformly bounded representations of the two by two unimodular group over local fields, Amer. J. Math., 90 (1968), 406-443.

29. P. J. Sally, Jr. and M. Taibleson, Special functions on locally compact fields, Acta Math., 116 (1966), 279-309.

30. G. Schiffmann, Integrales d'entrelacement et founctions de Whittaker, Bull. Soc. Math. France, 99 (1971), 3-72.

31. A. Silberger, On the work of MacDonald and $\left.L^{2} G / B\right)$ for a p-adic group, in Harmonic Analysis on Homogeneous Spaces, Proc. Symposia Pure Math., Amer. Math. Soc., Providence, R.I., (1973), 387-393.

32. - Introduction to harmonic analysis on reductive p-adic groups, to appear. 33. - The Knapp-Stein dimension theorem for p-adic groups, to appear.

34. R. Steinberg, Lectures on Chevalley Groups, Yale University Lecture Notes, New Heven, Conn., 1967.

35. M. Taibleson, Fourier Analysis on Local Fields, Princeton University Press, Princeton, 1975.

36. N. Winarsky, Reducibility of principal series representations of p-adic groups, thesis, University of Chicago, 1974.

37. - Reducibility of principal series representations of p-adic Chevalley groups, Amer. J. Math., 100 (1978), 941-956.

Received May 20, 1980.

UNIVERSITY OF UTAH

Salt Lake City, UT 84112 


\section{PACIFIC JOURNAL OF MATHEMATICS}

\section{EDITORS}

DONALD BABBITT (Managing Editor)

University of California

Los Angeles, CA 90024

Hugo Rossi

University of Utah

Salt Lake City, UT 84112

C. C. MOore and Arthur Agus

University of California

Berkeley, CA 94720
J. DugundJI

Department of Mathematics

University of Southern California

Los Angeles, CA 90007

R. FinN and J. MILGRAM

Stanford University

Stanford, CA 94305

\section{ASSOCIATE EDITORS}
R. ARENS
E. F, BECKENBACH
B. H. NeumanN
F. WOLF
K. YoSHIDA

\section{SUPPORTING INSTITUTIONS}

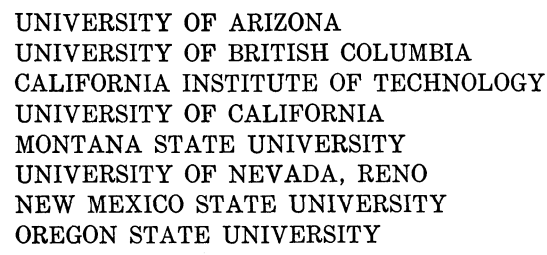

UNIVERSITY OF ARIZONA

UNIVERSITY OF BRITISH COLUMBIA

CALIFORNIA INSTITUTE OF TECHNOLOGY

UNIVERSITY OF CALIFORNIA

MONTANA STATE UNIVERSITY

UNIVERSITY OF NEVADA, RENO

NEW MEXICO STATE UNIVERSITY

OREGON STATE UNIVERSITY

\author{
UNIVERSITY OF OREGON \\ UNIVERSITY OF SOUTHERN CALIFORNIA \\ STANFORD UNIVERSITY \\ UNIVERSITY OF AAWAII \\ UNIVERSITY OF TOKYO \\ UNIVERSITY OF UTAH \\ WASHINGTON STATE UNIVERSITY \\ UNIVERSITY OF WASHINGTON
}

The Supporting Institutions listed above contribute to the cost of publication of this Journal, but they are not owners or publishers and have no responsibility for its content or policies,

Mathematical parers intended for publication in the Pacific Journal of Mathematics should be in typed form or offset-reproduced, (not dittoed), double spaced with large margins. Please do not use built up fractions in the text of the manuscript. However, you may use them in the displayed equations. Underline Greek letters in red, German in green, and script in blue. The first paragraph or two must be capable of being used separately as a synopsis of the entire paper. Please propose a heading for the odd unmbered pages of less than 35 characters. Manuscripts, in triplicate, may be sent to any one of the editors. Please classify according to the scheme of Math. Reviews, Index to Vol. 39. Supply name and address of author to whom proofs should be sent. All other communications should be addressed to the managing editor, or Elaine Barth, University of California, Los Angeles, California, 90024.

50 reprints to each author are provided free for each article, only if page charges have been substantially paid. Additional copies may be obtained at cost in multiples of 50 .

The Pacific Journal of Mathematics is issued monthly as of January 1966, Regular subscription rate: $\$ 114.00$ a year $(6$ Vol., 12 issues). Special rate: $\$ 57.00$ a year to individual members of supporting institution.

Subscriptions, orders for numbers issued in the last three calendar years, and changes of address shoud be sent to Pacific Journal of Mathematics, P.O. Box 969, Carmel Valley, CA 93924, U.S.A. Old back numbers obtainable from Kraus Periodicals Co., Route 100, Millwood, NY 10546.

PUBLISHED BY PACIFIC JOURNAL OF MATHEMATICS, A NON-PROFIT CORPORATION

Printed at Kokusai Bunken Insatsusha (International Academic Printing Co., Ltd.). 8-8, 3-chome, Takadanobaba, Shinjuku-ku, Tokyo 160, Japan.

Copyright (C) 1982 by Pacific Journal of Mathematics Manufactured and first issued in Japan 


\section{Pacific Journal of Mathematics}

\section{Vol. 101, No. 2 December, 1982}

Jean Bourgain, A Hausdorff-Young inequality for $B$-convex Banach

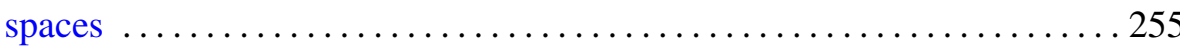

J. L. Brenner and Lorraine L. Foster, Exponential Diophantine

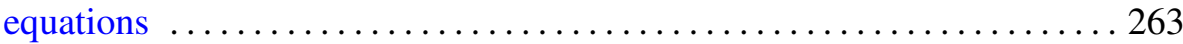

Henry H. Glover and William Duncan Homer, II, Fixed points on flag

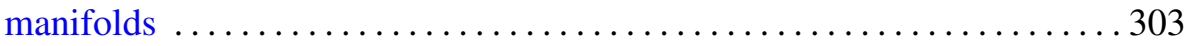

Lothar Hahn, A note on stochastic methods in connection with approximation theorems for positive linear operators $\ldots \ldots \ldots \ldots \ldots . \ldots 307$

James P. Henderson, Approximating cellular maps between low-dimensional polyhedra

V. K. Jain, Certain transformations of basic hypergeometric series and their

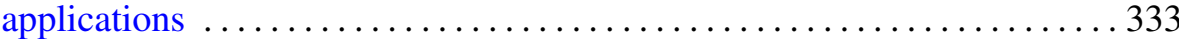

Charles David Keys, On the decomposition of reducible principal series

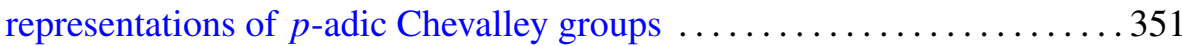

M. S. Klamkin and A. Meir, Ptolemy's inequality, chordal metric,

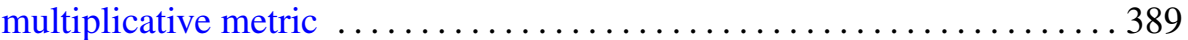

Robert F. Lax, Independence of normal Weierstrass points under

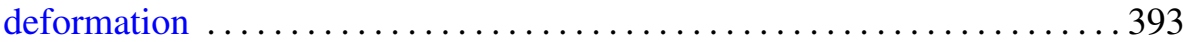

Leonid A. Luxemburg, On compactifications of metric spaces with

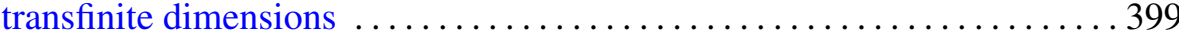

Carlton James Maxson, Martin Ross Pettet and Kirby C. Smith, On semisimple rings that are centralizer near-rings .....

Teodor C. Przymusiński, Extending functions from products with a metric factor and absolutes

Giorgio Talenti, A note on the Gauss curvature of harmonic and minimal surfaces

D. M. Terlinden, A spectral containment theorem analogous to the semigroup theory result $e^{t \sigma(A)} \subseteq \sigma\left(e^{t A}\right)$ 